Article

\title{
Next-Generation Sequencing Reveals the Role of Epigallocatechin-3-Gallate in Regulating Putative Novel and Known microRNAs Which Target the MAPK Pathway in Non-Small-Cell Lung Cancer A549 Cells
}

\author{
Vaishali Bhardwaj and Abul Kalam Azad Mandal * \\ Department of Biotechnology, School of Bio Sciences and Technology, Vellore Institute of Technology, \\ Tamil Nadu, Vellore 632014, India; shaali235@gmail.com \\ * Correspondence: akamandal@rediffmail.com; Tel.: +91-9443-758-596
}

Received: 21 November 2018; Accepted: 9 January 2019; Published: 21 January 2019

\begin{abstract}
Lung cancer constitutes $85 \%$ of non-small cell lung cancer diagnosed cases. MicroRNAs are novel biomarkers that are capable of modulating multiple oncogenic pathways. Epigallocatechin-3-gallate (EGCG) is a potent chemopreventive and chemotherapeutic agent for cancer. We aimed to identify important known and putative novel microRNAs modulated by EGCG in A549 cells using next-generation sequencing and identify their gene targets. Preliminary analysis revealed an IC50 value of $309 \mu \mathrm{M}$ with G0/G1 phase arrest at $40 \mu \mathrm{M}$ EGCG treatment. MicroRNA profiling identified 115 known and 4 putative novel microRNAs in $40 \mu \mathrm{M}$ and 134 known and 3 putative novel microRNAs in $100 \mu \mathrm{M}$ EGCG-treated A549 cells. The top 10 up-expressed microRNAs were similar between the untreated control and EGCG-treated A549 cells. An up-expression in oncogenic microRNAs, which belong to broadly conserved seed families, were observed in untreated control and EGCG-treated A549 cells. Kyoto Encyclopedia of Genes and Genomes and Protein Analysis Through Evolutionary Relationships pathway analyses of the validated microRNA targeting genes strengthened the hypothesis that EGCG treatment can modulate microRNAs that play a significant role in the MAPK signaling pathway. Expression profile of microRNAs was validation by quantitative real time PCR of randomly selected microRNAs. This study identified signature microRNAs that can be used as novel biomarkers for lung cancer diagnosis.
\end{abstract}

Keywords: next-generation sequencing; microRNAs; A549; EGCG; MAPK pathway

\section{Introduction}

Green tea, brewed from the unfermented dry leaves of the plant Camellia sinensis, is the most consumed non-alcoholic beverage in Asian countries and is now gaining popularity in western countries as well. It contains a wide range of phytochemicals which exhibits anti-cancer, anti-oxidative, and anti-inflammatory properties [1]. Among these phytochemicals, (-)-epigallocatechin-3-gallate (EGCG) accounts for $18-36 \%$ of the total phenolic compounds and $70 \%$ of the catechins present in green tea. Animal and cell line studies have demonstrated an important role of EGCG in promoting apoptosis and reducing cancer growth. EGCG is a potential chemopreventive and chemotherapeutic compound against skin [2], lung [3], breast [4], colon [5], prostate [6], and other cancers [7,8].

Lung cancer dominates among all the cancers with the leading mortality rate worldwide, $85 \%$ of which are non-small cell lung cancer (NSCLCs) cases [9]. Over $50 \%$ of the lung cancer patients die within a year of diagnosis with an estimated 1.6 million deaths per year [10]. The prevalent 
histological subtypes of NSCLCs include lung adenocarcinoma (LUAD), lung squamous cell carcinoma (LUSC) [11], and large cell lung cancer, out of which LUSC and large cell lung cancer are associated with smoking [12]. Clinical studies have showed that interaction between chemotherapeutic drugs and nicotine present in cigarette reduce the efficiency of chemotherapy in lung cancer patients by stimulating the cell survival pathways [13].

Despite many improvements in the field of early cancer diagnosis, the frequency of advanced stage detection is still notable. Tyrosine kinase inhibitors (TKIs) provide temporary benefits towards NSCLC resistance. However, EGFR (Epidermal growth factor receptor) mutations and ALK (Anaplastic lymphoma kinase) translocation are major challenges for TKIs in clinical practices [14]. Liquid biopsy study after drug therapy, radiological assessment as well as after and post-surgical adjuvant therapy explored circulating tumor cells (CTCs) and circulating tumor DNA (ctDNA) as useful biomarkers. These biomarkers are very useful to monitor TKIs which play an important role in detecting drug mutations [15]. Resistance development in cancer cells is the main challenge towards targeted therapy. The consensus analysis of liquid biopsies revealed CTCs and ctDNA/miRNA as important biomarkers for tumor profiling [15]. Thus, identification of novel diagnostic biomarkers and treatment approaches are the prerequisite for designing an optimal therapeutic regime. Recently, the cancer researchers have drifted their focus on the importance of microRNAs controlling expression of the target mRNAs which facilitates angiogenesis, tumor invasion, and growth [16,17].

Signaling pathways namely the RAS/RAF/MAPK [18], PI3K/AKT [19,20], JAK/STAT [21] and wnt/ $\beta$-Catenin [22] are very crucial for cancer growth and proliferation. The MAPK cascade plays an important role in human cancer cell survival, proliferation, and drug resistance [23]. It is an evolutionarily conserved, developmental pathway, which regulates tissue homeostasis and organ development. A transmitted signal cascade from receptor tyrosine kinases (RTKs), RAS and RAK family is relayed to important signaling molecules namely mitogen-activated extracellular signal-regulated kinase 1/2 (MAP2K1/2), and extracellular signal-regulated kinase 1/2 (MAPK3/1). Activated ERK1/2 nuclear translocation triggers transcriptional activation of many oncogenes, which modulates cellular processes responsible for cell proliferation, migration, angiogenesis, and cell survival [24]. Previous studies have identified a significant role of microRNAs in regulating MAPK signaling cascade in lung cancer $[25,26]$. The high prevalence rate of MAPK pathway in cancer regulation has motivated the oncology researchers in targeting critical pathway nodes of microRNAs and MAPK pathway.

MicroRNAs are a novel class of short, single-stranded, evolutionarily conserved non-coding RNA molecules (19-22 nucleotides in length), which play an important role in post-transcriptional gene regulation and modulation of biological processes including cell differentiation, proliferation, apoptosis, maintenance of cellular homeostasis etc. [27]. Extensive research on transcription regulation by microRNAs including oncogenes, proto-oncogenes and tumor suppressor genes [28-30] has explored the fundamental principles in the field of ncRNAs. Genome-scale mapping for microRNA profiling is a high throughput method for the identification of known and prediction of novel microRNAs [31]. MicroRNA profiling has validated that the altered expression of these microRNAs describes several pathologies including cancer [32-35] and these microRNAs can serve as a useful diagnostic and prognostic biomarker in lung cancer. The role of various microRNAs has been widely identified in lung cancer in which MAPK signaling cascade is known to play a significant role.

In the present study, we aimed to analyze the effect of EGCG on known as well as putative novel microRNAs in A549 cells and identify their gene targets for understanding their role in cancer pathways. Furthermore, qRT-PCR validation of selected microRNAs was performed to endorse our next-generation sequencing (NGS) data. 


\section{Results}

\subsection{EGCG Induced G0/G1 Phase Arrest in A549 Cell Line}

EGCG treatments significantly increased the percentage of cells at the G0/G1 phase of the cell cycle (Figure 1). The percentage of cells at the G0/G1 phase of the cell cycle increased from untreated control (55.58\%) to $40 \mu \mathrm{M}$ EGCG treatment $(79.15 \%)$. Furthermore, we observed that 79.15, 70.76, 78.76, and $72.04 \%$ of cells were persistent at the G0/G1 phase of the cell cycle at 40,60, 80, and $100 \mu \mathrm{M}$ EGCG treatments, respectively. A rational cell percentage at the G0/G1 phase of the cell cycle beyond $40 \mu \mathrm{M}$ EGCG treatment attests to G0/G1 phase arrest at $40 \mu \mathrm{M}$ EGCG treatment (Figure 1). In addition, no apoptosis was observed in EGCG-treated A549 cells.
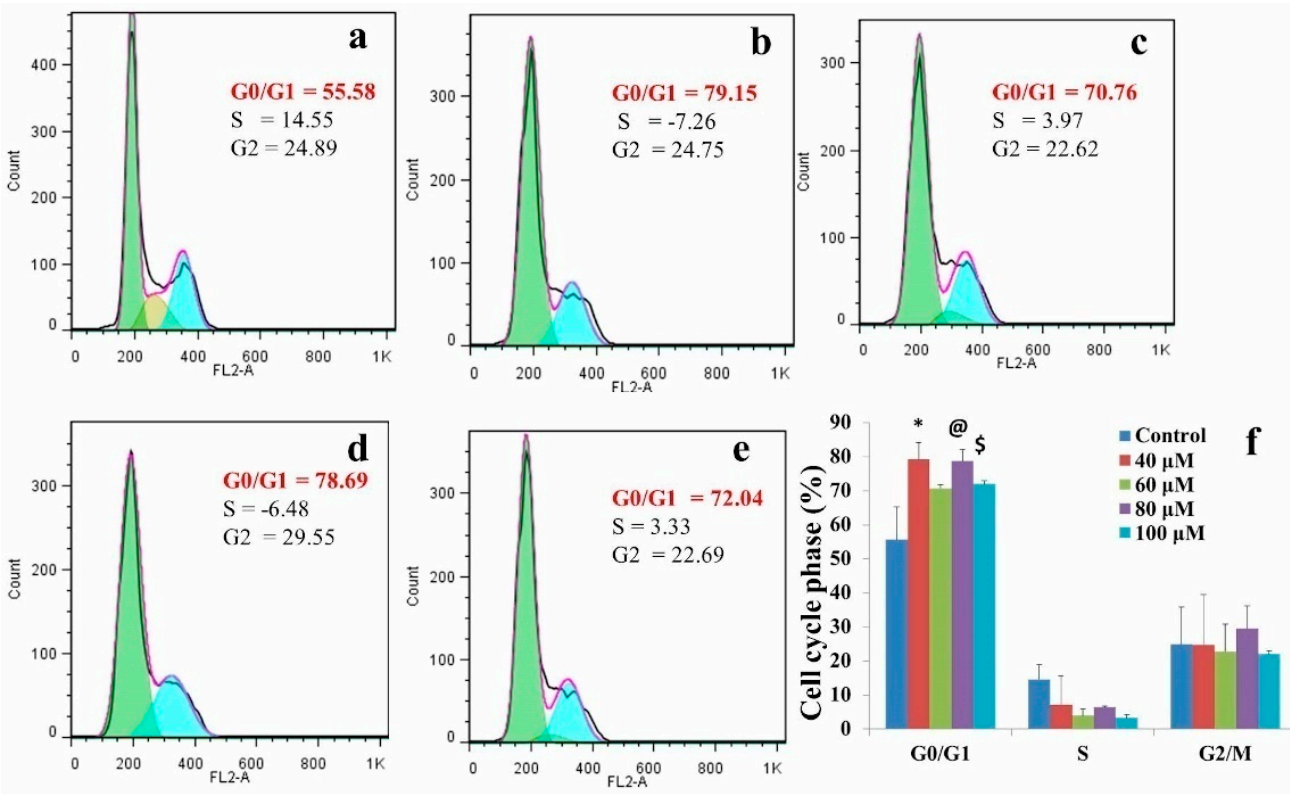

Figure 1. EGCG induced arrest at the G0/G1 phase of the cell cycle in A549 cells. (a) Untreated control (b) $40 \mu \mathrm{M}$ EGCG treatment (c) $60 \mu \mathrm{M}$ EGCG treatment (d) $80 \mu \mathrm{M}$ EGCG treatment and (e) $100 \mu \mathrm{M}$ EGCG treatment (f) the cell cycle distribution analysis. The data are shown as mean $\pm \mathrm{SD}$. Significant $(p<0.05)$ difference between the groups are indicated by: "** between untreated control and $40 \mu \mathrm{M}$ EGCG treatment, “@” between untreated control and $80 \mu \mathrm{M}$ EGCG treatment, and “\$” between untreated control and $100 \mu \mathrm{M}$ EGCG treatment.

\subsection{Analysis of MicroRNAs}

The miRBase-21 database was used for known miRNA detection using sequence similarity approach (ncbi-blast-2.2.30). Novel microRNA sequences were predicted using Mireap_0.22b [36]. In total, 958, 944 and 935 known microRNAs were detected in the untreated control, 40, and $100 \mu \mathrm{M}$ EGCG treatments, respectively. MicroRNAs with $\geq 50$ read count was counted as 206, 194, and 199 for untreated control, 40, and $100 \mu \mathrm{M}$ EGCG treatments, respectively. In addition, on an average, maximum microRNAs were predicted from chromosome 1 followed by chromosome 17 and 14 (Figure 2). About 105, 108, and 111 microRNAs were predicted from chromosome 1 followed by 82, 77, and 75 from chromosome 17 in the untreated control, 40, and $100 \mu$ M EGCG treatments, respectively. 


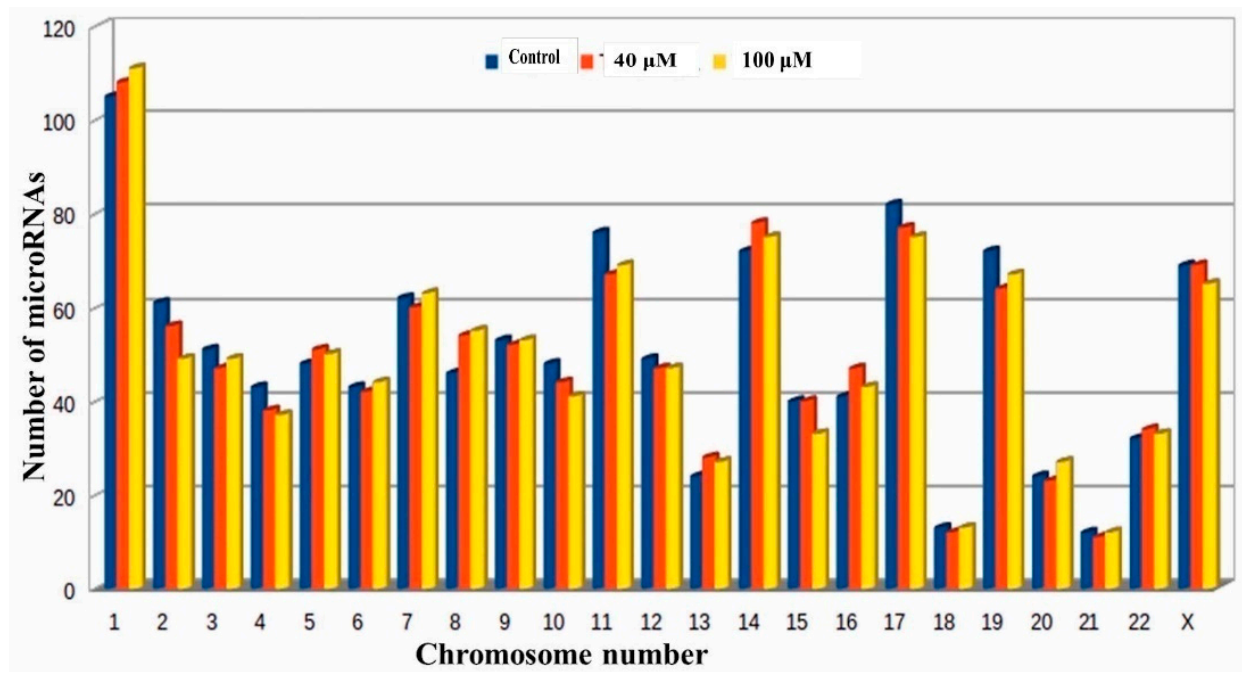

Figure 2. Chromosomal distribution of known microRNAs in the untreated control, 40 and $100 \mu \mathrm{M}$ EGCG treatments. Color key- blue: untreated control A549 cells, red: $40 \mu \mathrm{M}$ EGCG treatment and yellow: $100 \mu \mathrm{M}$ EGCG treatment.

\subsection{A549 Cell Associated MicroRNA Expression Signature}

We identified top ten up-expressed microRNAs (hsa-miR-21-5p, hsa-let-7i-5p, hsa-miR-100-5p, hsa-miR-27b-3p, hsa-miR-151a-3p, hsa-miR-148a-3p, hsa-miR-30a-5p, hsa-miR-192-5p, hsa-miR-3529-3p, and hsa-miR-30d-5p) in untreated control, 40 and $100 \mu \mathrm{M}$ EGCG treatments by the integrated analysis (Figure 3). Hsa-miR-21-5p was significantly up-expressed in the untreated control, 40, and $100 \mu \mathrm{M}$ EGCG treatments with the read count of 1403229, 1511476, and 1557436 respectively, followed by hsa-miR-7i-5p with 202380, 238919, and 231472 read counts in the untreated control, 40, and $100 \mu \mathrm{M}$ EGCG treatments, respectively. Surprisingly, these ten up-expressed microRNAs showed consistent up-regulation in the untreated control vs. 40 and the untreated control vs. $100 \mu \mathrm{M}$ EGCG treatments (Figure 3). The microRNA sequencing data indicated that top ten up-expressed microRNAs expression was not affected by EGCG treatment. Majority of these signature microRNAs belonged to the broadly conserved seed families namely let-7, hsa-miR-21, and hsa-miR-30.

\subsection{Prediction of Putative Novel MicroRNAs}

We identified top ten up-expressed predicted putative novel microRNA sequences in control, 40, and $100 \mu \mathrm{M}$ EGCG treatments and named as TPP-A549-1, TPP-A549-2 and so on. The chromosomal location, precursor, and mature sequences of the predicted putative novel miRNAs are presented in Table 1. We observed six putative novel microRNA sequences which were expressed in more than one sample. The predicted secondary structure of these putative novel microRNAs is presented in Figure 4. The putative novel microRNA TPP-A549-7 was expressed in all the samples with the MFE (Minimum free energy) value of $-29 \mathrm{Kcal} / \mathrm{mol}$ (Table 1). In the untreated control, the putative novel microRNA TPP-A549-1 was highly expressed with the read count of 3852. In addition, the putative novel microRNAs TPP-A549-11 and TPP-A549-17 were up-expressed in 40 and $100 \mu \mathrm{M}$ EGCG treatments with the read count of 998 and 1334 respectively (Table 1). 


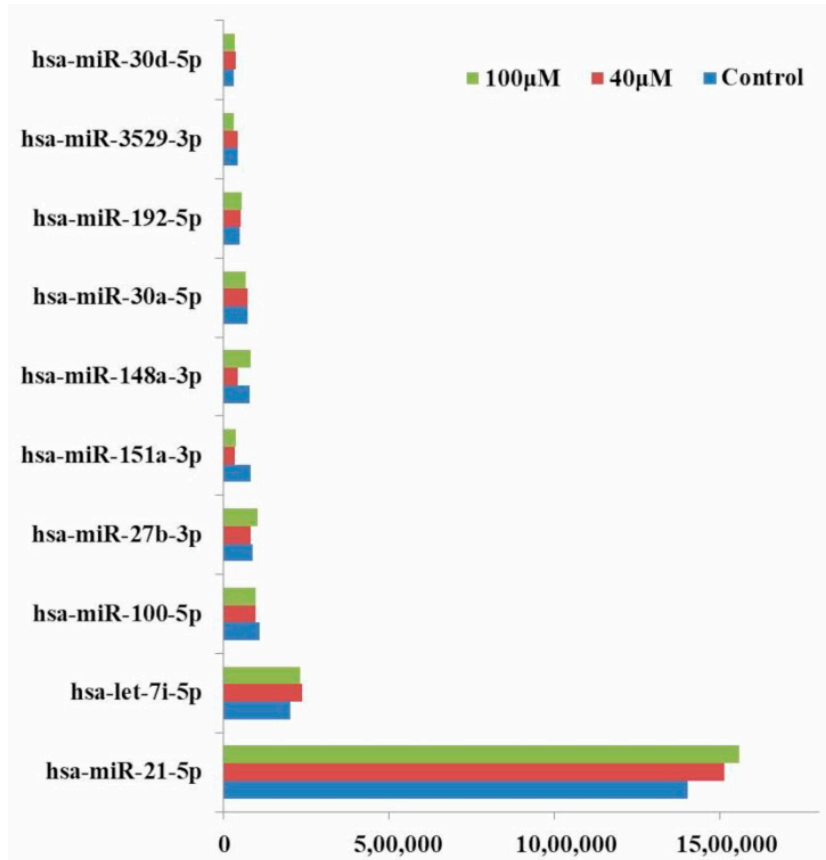

Figure 3. Read count of top ten microRNAs expressed in A549 cells after 40 and $100 \mu \mathrm{M}$ EGCG treatments.

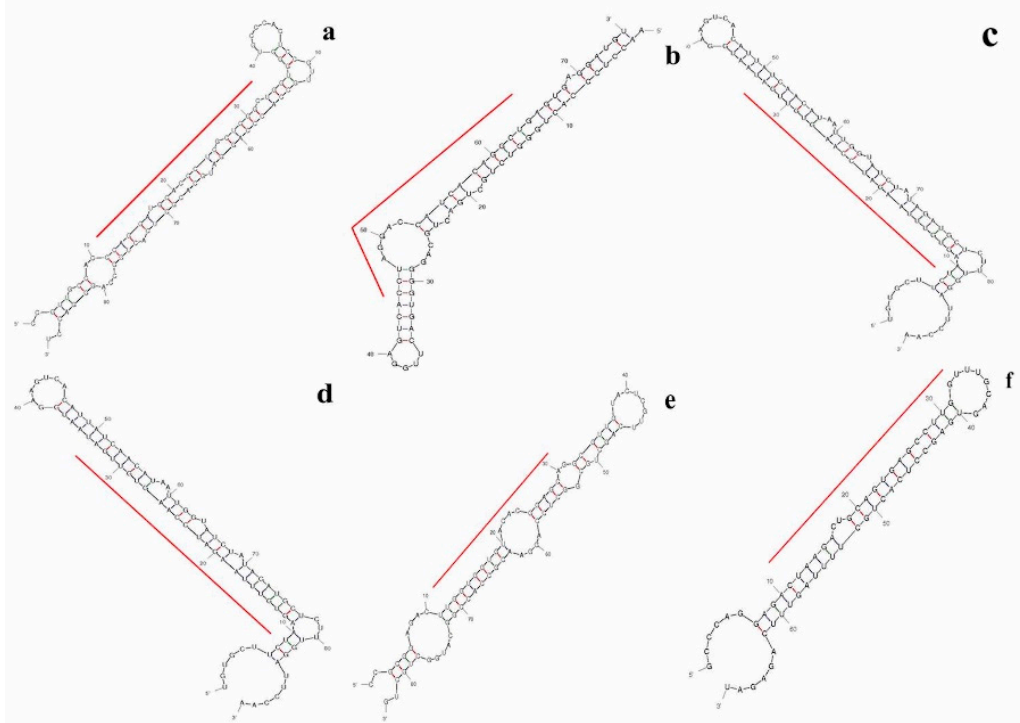

Figure 4. Predicted secondary structures of selected putative pri-miRNA sequences in the untreated control, 40, and $100 \mu \mathrm{M}$ EGCG treatments. The red line signifies the location of the putative mature microRNA sequence in the secondary structure. (a) CCAGGAUGCACGCUCGCUGGGCU; (b) CCUAGGACCAUCACAGGCUGA; (c) CAGAUGGUUGCUGAUCUGUGCA; (d) AGUGUUU AAGAUCCAAGUGUUG; (e) UUGGUGGUGUACACGGAGCAG; (f) CUAAGACUGCAGUGAG CCUUGGU. 
Table 1. Top 10 putative microRNA sequences expressed in the untreated control, 40, and $100 \mu \mathrm{M}$ EGCG treatments.

\begin{tabular}{|c|c|c|c|c|c|c|c|c|}
\hline $\begin{array}{l}\text { Putative Novel } \\
\text { MicroRNA ID }\end{array}$ & Sequences & $\begin{array}{l}\text { Chromosome } \\
\text { Number }\end{array}$ & Precursor Start & Precursor End & Mature Start & Mature End & Precursor Sequence & MFE (Kcal/mol) \\
\hline \multicolumn{9}{|c|}{ A549 Control } \\
\hline TPP-A549-1 & CCAGGAUGCACGCUCGCUGGGCU & 17 & 375,2068 & 375,2154 & 375,2078 & 375,2100 & $\begin{array}{l}\text { CCGUUGCCACCCAGGAUGCACGCUCGCUGGGCUGGUGGGUG } \\
\text { CCCACUCCGUUGCCACCCAGGAUGCACGCUCACUGGCCAGGG } \\
\text { ACCU }\end{array}$ & -30.7 \\
\hline TPP-A549-2 & CGCUUCGUGUAGACCCUCCAC & 15 & $748,733,11$ & $748,733,96$ & $748,733,21$ & $748,733,41$ & $\begin{array}{c}\text { GACCCAGCGGCGCUUCGUGUAGACCCUCCACUUCCGGGAGCG } \\
\text { AGGCAGCGGUUCUGGCGCAGGCGCGAUGCCCUCCCCCGAGG } \\
\text { GCG }\end{array}$ & -35.4 \\
\hline TPP-A549-3 & CUUAGAAUUCCGAUGCUGGGG & 22 & $267,618,39$ & $267,619,28$ & $267,618,98$ & $267,619,18$ & $\begin{array}{l}\text { AGAUUCUGGACUUAGAAUUCCGAUGCUGGGGCCAGGCACAG } \\
\text { UGGCUCACACCUGUAAUCCCAGUACUUUGGGAAGCCGAGGC } \\
\text { GGGAGGAU }\end{array}$ & -28.8 \\
\hline TPP-A549-4 & AGGCUGUAAGGCCACACGGGGUG & 18 & $374,244,03$ & $374,244,95$ & $374,244,63$ & $374,244,85$ & $\begin{array}{l}\text { CCUCCAAGGCAGGCUGUAAGGCCACACGGGGUGGAAGGCC } \\
\text { AGAGCUCCUUCUCCUCCUUCUGCUCUCUGGGUAUUGCCACCA } \\
\text { AGCCCUGUACC }\end{array}$ & -32.8 \\
\hline TPP-A549-5 & CCUAGGACCAUCACAGGCUGA & 16 & $888,546,38$ & $888,547,12$ & $888,546,82$ & $888,547,02$ & $\begin{array}{l}\text { AACCUCCCACUGGGUCUGCUGACUGCAGGGGUGACUUGGAG } \\
\text { UCACCUAGGACCAUCACAGGCUGAGUGAGGAUGU }\end{array}$ & -34.3 \\
\hline TPP-A549-6 & AUACUAUCUAGCUUUGGGUAGUA & 6 & $705,117,52$ & $705,118,32$ & $705,117,62$ & $705,117,84$ & $\begin{array}{l}\text { GCGUAGGAGUAACAGGCUGUAUUAUCUAGUUUAGGUGUGUA } \\
\text { GCAGGCUAUACUAUCUAGCUUUGGGUAGUAUAUUCUAUGA }\end{array}$ & -25.3 \\
\hline TPP-A549-7 & CAGAUGGUUGCUGAUCUGUGCA & 4 & $107,393,112$ & $107,393,209$ & $107,393,122$ & $107,393,143$ & $\begin{array}{l}\text { CAGGGUGUCCCAGAUGUCUCUGCAGCUUCCUCCUUGCAUCA } \\
\text { GAUGCCAGCAUCCAGUGACUCGAUCCAGAUGGUUGCUGAUCU } \\
\text { GUGCAGCAGAUCCUC }\end{array}$ & -29 \\
\hline TPP-A549-8 & AGUGUUUAAGAUCCAAGUGUUG & 4 & $911,143,10$ & $911,143,99$ & $911,143,68$ & $911,143,89$ & $\begin{array}{l}\text { UGUGCUUCUAAGUGUUUAAGAUCCAAGUGUUGAUAAUGGAAG } \\
\text { UCACAUUAUCAACAUAAUUGGUAUCUAUAGAUGCUCUUUGGA } \\
\text { UUCCAA }\end{array}$ & -29.2 \\
\hline TPP-A549-9 & UUGGUGGUGUACACGGAGCAG & 19 & $385,161,25$ & $385,162,07$ & $385,161,77$ & $385,161,97$ & $\begin{array}{l}\text { CCGCGGAGACUUGGUGGUGUACACGGAGCAGGCGUUGUACUC } \\
\text { GUUCAGCUGCGGCUCCAGGAACGCCACCGGCAUGGCUGCUG }\end{array}$ & -36.6 \\
\hline TPP-A549-10 & CCAGGACUCGAACCCUCCACCC & 8 & $144,458,110$ & $144,458,200$ & $144,458,169$ & $144,458,190$ & $\begin{array}{l}\text { ACAUGGGUCUGAGAGGGACUGGCACGUUGUCCAAGGCCGCAC } \\
\text { AGCUGUUAAGCAUGAAGCCAGGACUCGAACCCUCCACCCAGGC } \\
\text { UGGAUU }\end{array}$ & -29.8 \\
\hline \multicolumn{9}{|c|}{$40 \mu \mathrm{M}$ EGCG Treatment } \\
\hline TPP-A549-11 & CUAAGACUGCAGUGAGCCUUGGU & 16 & 589,8984 & 589,9051 & 589,8994 & 589,9016 & $\begin{array}{l}\text { GCCCAGGAGACUAAGACUGCAGUGAGCCUUGGUUUGCAGUGA } \\
\text { GCCUCACUGCUUUUAGUUUCAGAGAU }\end{array}$ & -25.1 \\
\hline TPP-A549-12 & CACCUGUAGUUCCAUCUACUCCAG & 7 & $789,447,92$ & 789,44892 & $789,448,59$ & $789,448,82$ & $\begin{array}{l}\text { UAGUGGCGUGCACCUGUAGUUCCAUCUACUCCAGAGGCUGAG } \\
\text { GUGGGGGGAUCGUUUGAGCUUGGGAGAUGGAGGUUGCAGUGA } \\
\text { GCAGUGAUUGUGCCACU }\end{array}$ & -38.51 \\
\hline TPP-A549-13 & CAAGCCAUUGAUCCCAAAGUCA & 1 & $535,110,24$ & $535,111,16$ & $535,110,85$ & $535,111,06$ & $\begin{array}{l}\text { CCAGAGCCCCCAAGCCAUUGAUCCCAAAGUCAUUUUAGCCCCU } \\
\text { GUGAACGGGGCCCAGGGGCUUAGGAAGGUAGCAUGAGAAGGG } \\
\text { AACCUGGC }\end{array}$ & -29.3 \\
\hline TPP-A549-14 & CUCUGAACUGGGUAGAUCUGUC & 2 & $181,190,09$ & $181,190,98$ & $181,190,19$ & $181,190,40$ & $\begin{array}{l}\text { AACAGGGUAGCUCUGAACUGGGUAGAUCUGUCUGUCAAGAGG } \\
\text { AACGCUCUAGAAAGCUCAGGACUCUCCUGCACAGGGCUUAGC } \\
\text { CCACUG }\end{array}$ & -31.6 \\
\hline TPP-A549-9 & UUGGUGGUGUACACGGAGCAGGC & 19 & $385,161,25$ & $385,162,07$ & 3851,6175 & $385,161,97$ & $\begin{array}{l}\text { CCGCGGAGACUUGGUGGUGUACACGGAGCAGGCGUUGUACUC } \\
\text { GUUCAGCUGCGGCUCCAGGAACGCCACCGGCAUGGCUGCUG }\end{array}$ & -36.6 \\
\hline
\end{tabular}


Table 1. Cont.

\begin{tabular}{|c|c|c|c|c|c|c|c|c|}
\hline $\begin{array}{l}\text { Putative Novel } \\
\text { MicroRNA ID }\end{array}$ & Sequences & $\begin{array}{l}\text { Chromosome } \\
\text { Number }\end{array}$ & Precursor Start & Precursor End & Mature Start & Mature End & Precursor Sequence & MFE (Kcal/mol) \\
\hline TPP-A549-8 & AGUGUUUAAGAUCCAAGUGUUG & 4 & $911,143,10$ & $911,143,99$ & $911,143,68$ & $911,143,89$ & $\begin{array}{l}\text { UGUGCUUCUAAGUGUUUAAGAUCCAAGUGUUGAUAAUGGAA } \\
\text { GUCACAUUAUCAACAUAAUUGGUAUCUAUAGAUGCUCUUUGG } \\
\text { AUUCCAA }\end{array}$ & -29.2 \\
\hline TPP-A549-7 & CAGAUGGUUGCUGAUCUGUGCA & 4 & $107,393,112$ & $107,393,209$ & $107,393,122$ & $107,393,143$ & $\begin{array}{l}\text { CAGGGUGUCCCAGAUGUCUCUGCAGCUUCCUCCUUGCAUCAG } \\
\text { AUGCCAGCAUCCAGUGACUCGAUCCAGAUGGUUGCUGAUCUG } \\
\text { UGCAGCAGAUCCUC }\end{array}$ & -29 \\
\hline TPP-A549-3 & CUAGGACCAUCACAGGCUGA & 16 & $888,546,38$ & $888,547,12$ & $888,546,83$ & $888,547,02$ & $\begin{array}{l}\text { AACCUCCCACUGGGUCUGCUGACUGCAGGGGUGACUUGGAGUC } \\
\text { ACCUAGGACCAUCACAGGCUGAGUGAGGAUGU }\end{array}$ & -34.3 \\
\hline TPP-A549-15 & AAUUUCUGAUCCUGGCCAUAUUC & 1 & 414,2086 & 414,2164 & 414,2096 & 414,2118 & $\begin{array}{l}\text { ACAGAGGAUAGAGUUGGGCAGCAUCAGAAAGCCCAGCUUCCUC } \\
\text { UGGAAUUUCUGAUCCUGGCCAUAUUCACUUCCUGUG }\end{array}$ & -34.5 \\
\hline TPP-A549-16 & GCUAAAACUGGUCAAAGUGCUG & 6 & $117,578,916$ & $117,579,011$ & $117,578,926$ & $117,578,947$ & $\begin{array}{l}\text { UGUUUUGGAGAAAGAAGUACAUGAUCAGCUUUUACAGCUGCAC } \\
\text { UCUAUUCAGCUGCAGCUUCAUGCUAAAACUGGUCAAAGUGCUG } \\
\text { ACUCUGGUAC }\end{array}$ & -28.5 \\
\hline \multicolumn{9}{|c|}{$100 \mu \mathrm{M}$ EGCG Treatment } \\
\hline TPP-A549-17 & AUCAGUGGUUCUCAAUGUUCUUUU & 1 & 797,2080 & 797,2153 & 797,2120 & 797,2143 & $\begin{array}{l}\text { GUUAUGGGCCAGAGGUCCUGGGGACUUGAUACAUUCGAGUAUC } \\
\text { AGUGGUUCUCAAUGUUCUUUUGGUGCACUUG }\end{array}$ & -27.5 \\
\hline TPP-A549-11 & CUAAGACUGCAGUGAGCCUUGGU & 16 & 589,8984 & 589,9051 & 589,8994 & 589,9016 & $\begin{array}{l}\text { GCCCAGGAGACUAAGACUGCAGUGAGCCUUGGUUUGCAGUGAG } \\
\text { CCUCACUGCUUUUAGUUUCAGAGAU }\end{array}$ & -25.1 \\
\hline TPP-A549-18 & CAUCCACCUGGUCCAGCUCGC & 16 & 317,07819 & $317,079,08$ & $317,078,29$ & $317,078,49$ & $\begin{array}{l}\text { ACCUGGGAGGGACCAAGGCUGGGAGGGUGAGAGGCCAUGCCCA } \\
\text { CAAAAGCCUCAGGCUACAUCCACCUGGUCCAGCUCGCCAAGA } \\
\text { UGGGA }\end{array}$ & -34.2 \\
\hline TPP-A549-19 & GCUGUUGUUGCUGUGGUUGUGGCU & 2 & 182,3300 & 182,3382 & 182,3310 & 182,3333 & $\begin{array}{l}\text { UGGCUGUGAGGCUGUUGUUGCUGUGGUUGUGGCUGCAACCUU } \\
\text { CAUUGUCAUAGAUAUAGCUGUGGCAGCUGUAGUUGUAGCCU }\end{array}$ & -34.02 \\
\hline TPP-A549-7 & CAGAUGGUUGCUGAUCUGUGCA & 4 & $107,3931,12$ & $107,393,209$ & $107,393,122$ & $107,393,143$ & $\begin{array}{l}\text { CAGGGUGUCCCAGAUGUCUCUGCAGCUUCCUCCUUGCAUCAGA } \\
\text { UGCCAGCAUCCAGUGACUCGAUCCAGAUGGUUGCUGAUCUGU } \\
\text { GCAGCAGAUCCUC }\end{array}$ & -29 \\
\hline TPP-A549-20 & GCUUAGCUAAGAAUACUUAUAAUU & 3 & $997,767,29$ & $997,768,24$ & $997,767,39$ & $997,767,62$ & $\begin{array}{l}\text { AGCUUGGGCUGCUUAGCUAAGAAUACUUAUAAUUGUUUCUUG } \\
\text { AUUAUAUGCUAAACAAGGGGUGGAUUCAUGAGUUUUCUGGG } \\
\text { AAAGGGGUAGGCA }\end{array}$ & -25.5 \\
\hline TPP-A549-21 & GAAGCUGUUGGACUAGAAAAAAUU & 4 & $106,688,766$ & $106,688,864$ & $1066,888,31$ & $106,688,854$ & $\begin{array}{c}\text { UACCCUCUGAUAGGUUUUCUCAGCUGCAGCUUCACUGUGUGC } \\
\text { AUAACCUCACUGUGGGGCUCAAGGAAGCUGUUGGACUAGA } \\
\text { AAAAAUUUCAUUGCUGC }\end{array}$ & -25.6 \\
\hline TPP-A549-1 & CCAGGAUGCACGCUCGCUGGGC & 17 & 375,2068 & 375,2154 & 375,2078 & 375,2099 & $\begin{array}{l}\text { CCGUUGCCACCCAGGAUGCACGCUCGCUGGGCUGGUGGGUG } \\
\text { CCCACUCCGUUGCCACCCAGGAUGCACGCUCACUGGCCAG } \\
\text { GGACCU }\end{array}$ & -30.7 \\
\hline TPP-A549-22 & GUAAAGACGUUGAUGCUGCUA & 17 & 857,0265 & 857,0362 & 857,0332 & 857,0352 & $\begin{array}{l}\text { AGGAGUUUCAUAUACUAUCUCUAUCAUAUAGACAGGGCAA } \\
\text { UGUCUUCACCUCCACUUUCAGAGGAUGGUAAAGACGUUG } \\
\text { AUGCUGCUAACUUGCUCAG }\end{array}$ & -26.22 \\
\hline TPP-A549-26 & UCUUCAUCUGGUUUGUGAACUUUU & 17 & $569,836,22$ & $569,837,13$ & $569,836,32$ & $569,836,55$ & $\begin{array}{l}\text { GCGGUUAACAUCUUCAUCUGGUUUGUGAACUUUUUACUGC } \\
\text { UUAGGAAAUCUUAAGAUACAAAGGGCAUAUGAUCAGGGGG } \\
\text { AUGUAUAGCCUU }\end{array}$ & -31.7 \\
\hline
\end{tabular}




\subsection{Differential Expression Analysis of Known MicroRNAs}

A complete microRNA profiling is depicted in Figure 5, indicating the effect of EGCG on A549 cells. MicroRNA expression with greater than $2 \log 2$ fold change was determined in the untreated control vs. $40 \mu \mathrm{M}$ and the untreated control vs. $100 \mu \mathrm{M}$ EGCG treatments. The heat maps of the top 100 differentially expressed microRNAs between the samples are presented in Figure 6a,b.

A complete list of up- and down-regulated microRNAs is presented in Table 2. We found 115 microRNAs differentially expressed in the untreated control vs. $40 \mu \mathrm{M}$ EGCG treatment, and 134 microRNAs differentially expressed in control vs. $100 \mu \mathrm{M}$ EGCG treatment (Figure 6c). Out of the 115 differentially expressed microRNAs reported in the untreated control vs. $40 \mu \mathrm{M}$ EGCG treatment, 53 were up- and 62 were down-regulated. Furthermore, in the untreated control vs. $100 \mu \mathrm{M}$ EGCG treatment, we reported 69 up- and 65 down-regulated microRNAs (Figure 6d).

By comparing the data with all the reported up-regulated microRNAs, hsa-miR-125a-3p showed the highest change of $\log 2$ fold expression in the untreated control vs. $40 \mu \mathrm{M}$ EGCG treatment $(7.12 \log 2$ fold change) and untreated control vs. $100 \mu \mathrm{M}$ EGCG treatment (7.47 log2 fold change). Furthermore, hsa-miR-548o-3p was down-regulated by -9.12 and $-8.12 \log 2$ fold change in the untreated control vs. $40 \mu \mathrm{M}$ EGCG and the untreated control vs. $100 \mu \mathrm{M}$ EGCG treatments, respectively. We observed 21 up- and 24 down-regulated microRNAs in the untreated control vs. 40 and the untreated control vs $100 \mu \mathrm{M}$ EGCG treatments (Figure 7). 


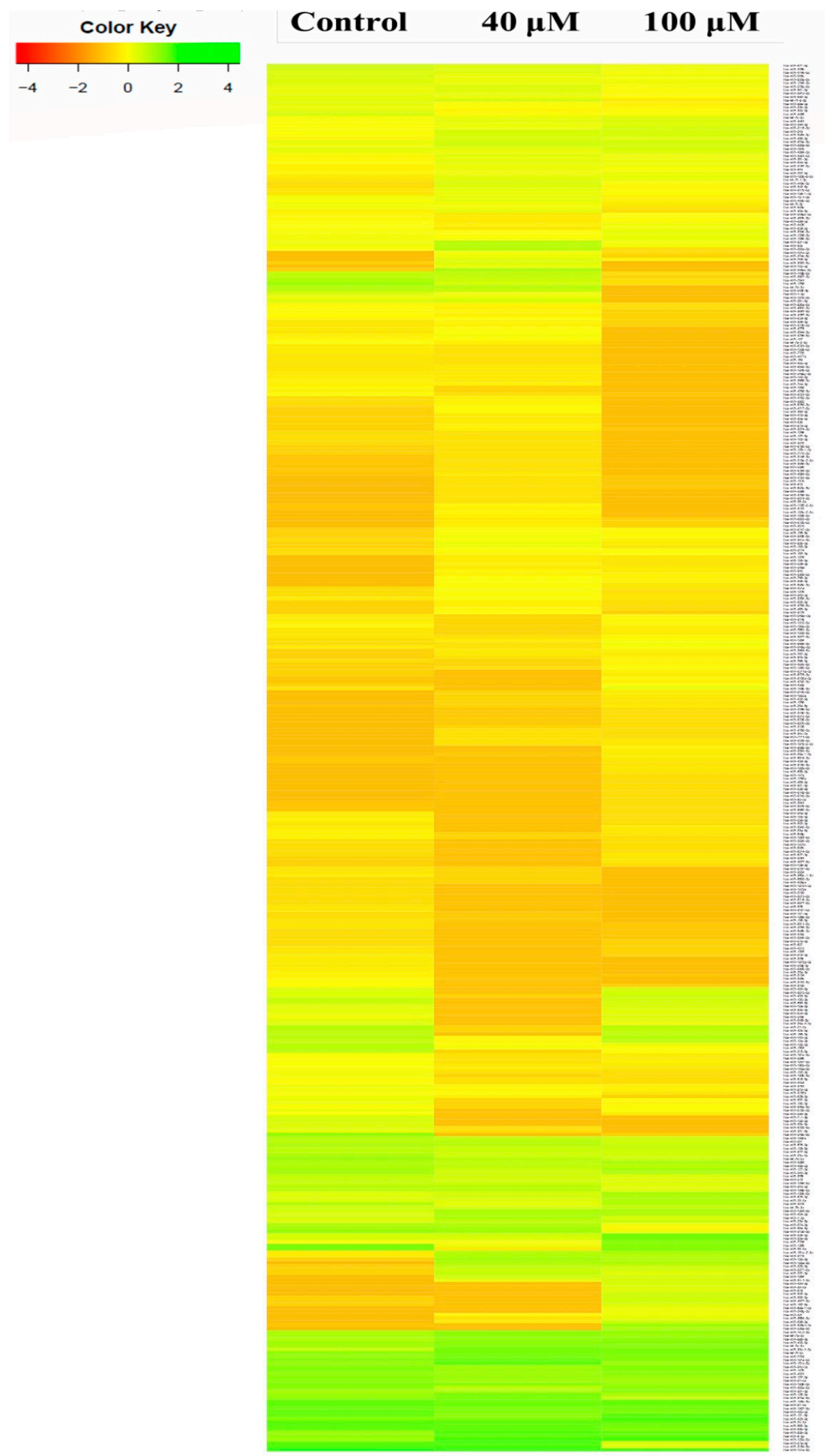

Figure 5. The expression profiles of known microRNAs in the untreated control, $40 \mu \mathrm{M}$, and $100 \mu \mathrm{M}$ EGCG treatments. Color key- red: up-regulation, green: down-regulation and yellow: neutral expression. 


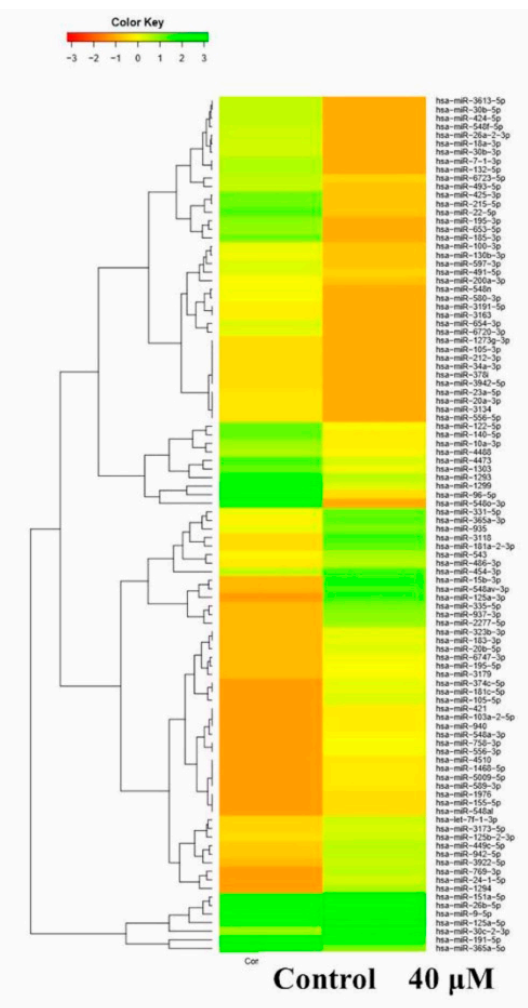

a

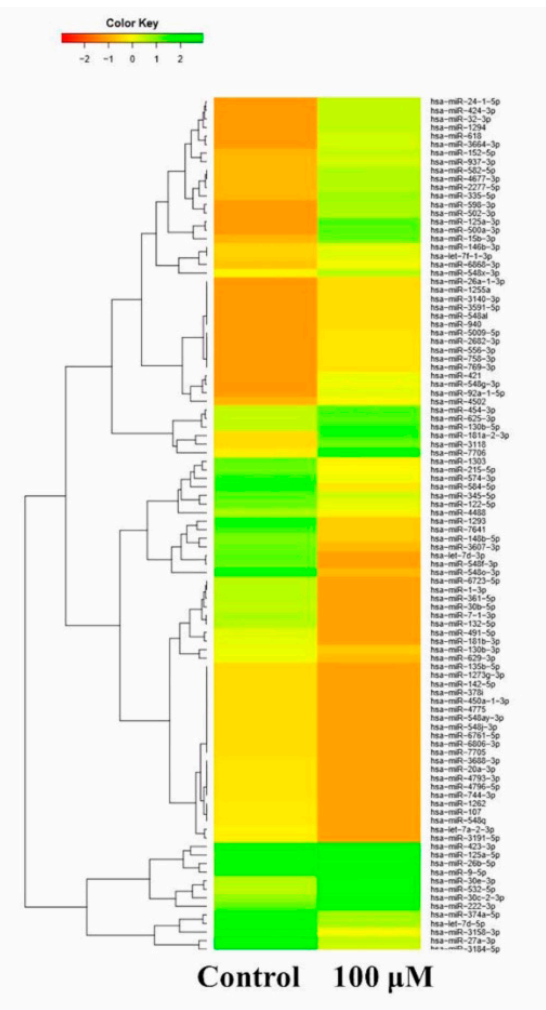

$\mathbf{b}$

d

\section{C vs $40 \mu \mathrm{M} C$ vs $100 \mu \mathrm{M} \quad 40$ vs $100 \mu \mathrm{M}$}
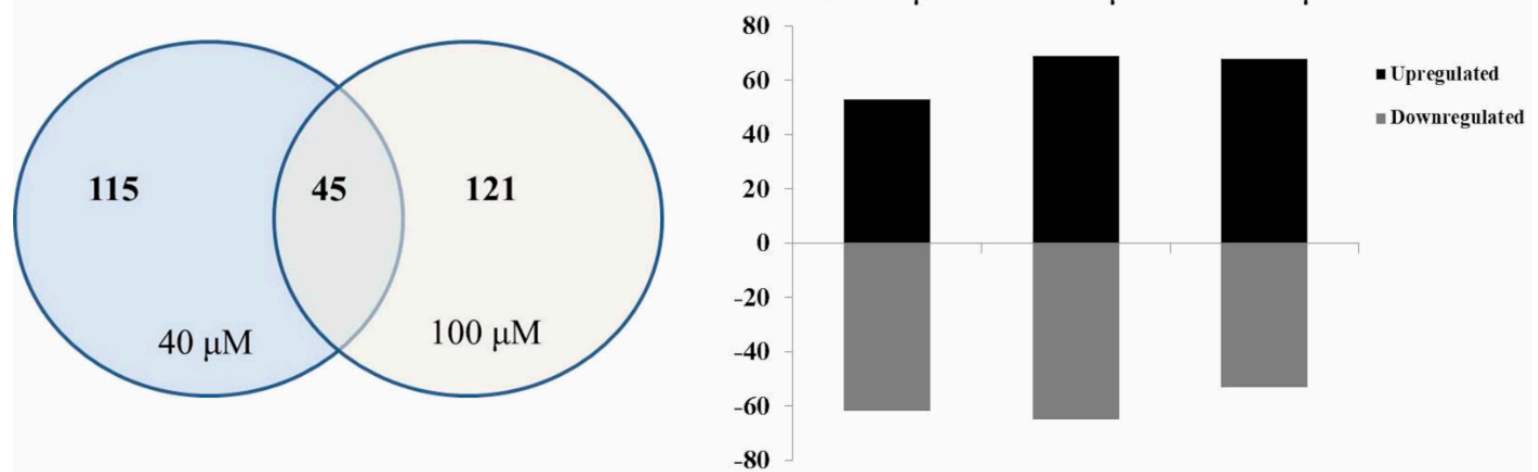

Figure 6. Dose-dependent microRNA expression profiles of top 100 microRNAs. (a) Relative microRNA expression levels in untreated control vs. $40 \mu \mathrm{M}$ EGCG treatment; (b) Relative microRNA expression levels in untreated control vs. $100 \mu \mathrm{M}$ EGCG treatment; (c) Venn diagram depicting the dose-dependent responses of microRNAs to EGCG; (d) Summary set of up- and down-regulated microRNAs exhibiting greater than $2 \log 2$-fold change expression after EGCG treatments. Color key- red: up-regulation, green: down-regulation and yellow: neutral expression. 
Table 2. List of known microRNAs with greater than $2 \log 2$ fold change expression after 40 and $100 \mu \mathrm{M}$ EGCG treatments.

\begin{tabular}{|c|c|c|}
\hline \multirow{2}{*}{ Treatments Compared } & \multirow{2}{*}{ No. of MicroRNAs } & \multirow[b]{2}{*}{ Up-Regulated } \\
\hline & & \\
\hline Control vs. $40 \mu \mathrm{M}$ & 115 & $\begin{array}{c}\text { hsa-miR-125a-3p, hsa-miR-15b-3p, hsa-miR-548av-3p, hsa-miR-1294, } \\
\text { hsa-miR-335-5p, hsa-miR-937-3p, hsa-miR-3118, hsa-miR-125a-5p, } \\
\text { hsa-miR-769-3p, hsa-miR-2277-5p, hsa-miR-30c-2-3p, hsa-miR-24-1-5p, } \\
\text { hsa-miR-181a-2-3p, hsa-miR-105-5p, hsa-miR-181c-5p, } \\
\text { hsa-miR-365a-3p, hsa-miR-374c-5p, hsa-miR-3922-5p, hsa-miR-26b-5p, } \\
\text { hsa-miR-449c-5p, hsa-miR-556-3p, hsa-miR-758-3p, hsa-miR-151a-5p, } \\
\text { hsa-miR-331-5p, hsa-miR-942-5p, hsa-miR-20b-5p, hsa-miR-548a-3p, } \\
\text { hsa-miR-103a-2-5p, hsa-miR-323b-3p, hsa-miR-421, hsa-miR-183-3p, } \\
\text { hsa-miR-940, hsa-miR-935, hsa-miR-9-5p, hsa-let-7f-1-3p, } \\
\text { hsa-miR-1468-5p, hsa-miR-4510, hsa-miR-5009-5p, hsa-miR-589-3p, } \\
\text { hsa-miR-6747-3p, hsa-miR-195-5p, hsa-miR-486-3p, hsa-miR-454-3p, } \\
\text { hsa-miR-3173-5p, hsa-miR-543, hsa-miR-125b-2-3p, hsa-miR-155-5p, } \\
\text { hsa-miR-3179, hsa-miR-1976, hsa-miR-598-3p, hsa-miR-548al, } \\
\text { hsa-miR-6853-3p, hsa-miR-6733-5p }\end{array}$ \\
\hline
\end{tabular}

hsa-miR-548ah-3p, hsa-miR-96-5p, hsa-miR-500a-3p, hsa-miR-185-3p, hsa-miR-22-5p, hsa-miR-7706, hsa-miR-30e-3p, hsa-miR-532-5p, hsa-miR-502-3p, hsa-miR-1299, hsa-miR-4677-3p, hsa-miR-582-5p, hsa-miR-195-3p, hsa-miR-424-5p, hsa-miR-425-3p, hsa-miR-3613-5p, hsa-miR-653-5p, hsa-miR-424-3p, hsa-miR-152-5p, hsa-miR-32-3p, hsa-miR-18a-3p, hsa-miR-548n, hsa-miR-493-5p, hsa-miR-30b-3p, hsa-miR-18a-3p, hsa-miR-548n, hsa-miR-493-5p, hsa-miR-30b-3p,
hsa-miR-618, hsa-miR-654-3p, hsa-miR-548f-5p, hsa-miR-140-5p hsa-miR-92a-1-5p, hsa-miR-146b-3p, hsa-miR-548g-3p,

hsa-miR-26a-2-3p, hsa-miR-10a-3p, hsa-miR-598-3p, hsa-miR-4502, hsa-miR-423-3p, hsa-miR-191-5p, hsa-miR-6720-3p, hsa-miR-3664-3p, hsa-miR-130b-5p, hsa-miR-3150b-3p, hsa-miR-4473, hsa-miR-2682-3p hsa-miR-365a-5p, hsa-miR-215-5p, hsa-miR-4742-3p,

hsa-miR-6511a-3p, hsa-miR-28-5p, hsa-miR-580-3p, hsa-miR-3942-5p, hsa-miR-6775-3p, hsa-miR-6818-3p, hsa-miR-3591-5p, hsa-miR-625-3p, hsa-miR-6868-3p, hsa-miR-597-3p, hsa-miR-381-3p, hsa-miR-127-3p, hsa-miR-1229-3p, hsa-miR-200a-3p, hsa-miR-23a-5p, hsa-miR-3120-3p,

hsa-miR-433-3p, hsa-miR-4677-5p, hsa-miR-655-3p, hsa-miR-5091,

croRNAs

Down-Regulated

hsa-miR-548o-3p, hsa-miR-96-5p, hsa-miR-185-3p, hsa-miR-1299 hsa-miR-22-5p, hsa-miR-195-3p, hsa-miR-653-5p, hsa-miR-425-3p, hsa-miR-215-5p, hsa-miR-7-1-3p, hsa-miR-132-5p, hsa-miR-424-5p, (a) hsa-miR-3613-5p, hsa-miR-30b-5p, hsa-miR-548f-5p, hsa-miR-26a-2-3p, hsa-miR-18a-3p, hsa-miR-30b-3p, hsa-miR-654-3p, hsa-miR-140-5p, hsa-miR-6720-3p, hsa-miR-493-5p, hsa-miR-122-5p, hsa-miR-548n, hsa-miR-580-3p, hsa-miR-6723-5p, hsa-miR-1303, hsa-miR-597-3p hsa-miR-1293, hsa-miR-10a-3p, hsa-miR-4473, hsa-miR-3163, hsa-miR-3191-5p, hsa-miR-365a-5p, hsa-miR-100-3p, hsa-miR-191-5p, hsa-miR-130b-3p, hsa-miR-20a-3p, hsa-miR-3134, hsa-miR-23a-5p, hsa-miR-556-5p, hsa-miR-4488, hsa-miR-491-5p, hsa-miR-378i,

hsa-miR-3942-5p, hsa-miR-548j-3p, hsa-miR-6806-3p, hsa-miR-200a-3p, hsa-miR-212-3p, hsa-miR-105-3p, hsa-miR-1273g-3p, hsa-miR-34a-3p, hsa-miR-342-3p, hsa-miR-192-3p, hsa-miR-29c-5p, hsa-let-7d-5p, hsa-miR-1305, hsa-miR-146b-3p, hsa-miR-578, hsa-miR-6811-5p, hsa-miR-552-3p, hsa-miR-4755-3p, hsa-miR-3127-5p

hsa-miR-3184-5p, hsa-miR-548av-3p, hsa-miR-27a-3p, hsa-let-7d-3p, hsa-miR-548f-3p, hsa-miR-361-5p, hsa-miR-365a-3p, hsa-miR-3607-3p, hsa-miR-584-5p, hsa-miR-3922-5p, hsa-miR-148b-5p, hsa-miR-7641, hsa-miR-181b-3p, hsa-miR-3158-3p, hsa-miR-105-5p, hsa-miR-4796-5p, hsa-miR-1-3p, hsa-miR-151a-5p, hsa-miR-374a-5p, hsa-miR-1293,

hsa-miR-4775, hsa-miR-574-3p, hsa-miR-331-5p, hsa-miR-107,

hsa-miR-4717-5p, hsa-miR-363-3p, hsa-let-7f-2-3p, hsa-miR-454-5p, hsa-miR-103a-2-5p, hsa-miR-374c-5p, hsa-miR-6783-3p,

hsa-miR-1185-2-3p, hsa-miR-935, hsa-miR-1468-5p, hsa-miR-744-3p hsa-miR-3131, hsa-miR-4762-3p, hsa-miR-873-5p, hsa-miR-3923, hsa-let-7a-2-3p, hsa-miR-769-3p, hsa-miR-1249-5p, hsa-miR-142-5p, hsa-miR-181c-5p, hsa-miR-3179, hsa-miR-33a-5p, hsa-miR-3688-3p hsa-miR-3944-3p, hsa-miR-410-3p, hsa-miR-4684-5p, hsa-miR-548ay-3p, hsa-miR-636, hsa-miR-653-3p, hsa-miR-6843-3p hsa-miR-627-3p, hsa-miR-548aj-5p 
Table 2. Cont

\begin{tabular}{|c|c|c|c|}
\hline \multirow{2}{*}{ Treatments Compared } & \multirow{2}{*}{ No. of MicroRNAs } & \multicolumn{2}{|c|}{ MicroRNAs } \\
\hline & & Up-Regulated & Down-Regulated \\
\hline Control vs. $100 \mu \mathrm{M}$ & 134 & $\begin{array}{c}\text { hsa-miR-125a-3p, hsa-miR-500a-3p, hsa-miR-7706, hsa-miR-15b-3p, } \\
\text { hsa-miR-598-3p, hsa-miR-532-5p, hsa-miR-502-3p, hsa-miR-30e-3p, } \\
\text { hsa-miR-181a-2-3p, hsa-miR-1294, hsa-miR-24-1-5p, hsa-miR-424-3p, } \\
\text { hsa-miR-335-5p, hsa-miR-32-3p, hsa-miR-125a-5p, hsa-miR-618, } \\
\text { hsa-miR-3664-3p, hsa-miR-3118, hsa-miR-582-5p, hsa-miR-4677-3p, } \\
\text { hsa-miR-2277-5p, hsa-miR-30c-2-3p, hsa-miR-152-5p, } \\
\text { hsa-miR-92a-1-5p, hsa-miR-421, hsa-miR-937-3p, hsa-miR-423-3p, } \\
\text { hsa-miR-26b-5p, hsa-miR-548g-3p, hsa-miR-130b-5p, hsa-miR-625-3p, } \\
\text { hsa-miR-9-5p, hsa-miR-556-3p, hsa-miR-758-3p, hsa-miR-769-3p, } \\
\text { hsa-miR-5009-5p } \\
\text { hsa-miR-2682-3p, hsa-miR-454-3p, hsa-miR-4502, hsa-miR-222-3p, } \\
\text { hsa-miR-26a-1-3p, hsa-miR-3140-3p, hsa-miR-3591-5p, hsa-miR-548al, } \\
\text { hsa-miR-940, hsa-miR-1255a, hsa-let-7f-1-3p, hsa-miR-146b-3p, } \\
\text { hsa-miR-6868-3p, hsa-miR-548x-3p, hsa-miR-1285-5p, } \\
\text { hsa-miR-1304-3p, hsa-miR-2116-3p, hsa-miR-28-5p, hsa-miR-543, } \\
\text { hsa-miR-1229-3p, hsa-miR-1290, hsa-miR-155-5p, hsa-miR-181b-2-3p, } \\
\text { hsa-miR-1976 } \\
\text { hsa-miR-3120-3p, hsa-miR-3129-5p, hsa-miR-3174, hsa-miR-323b-3p, } \\
\text { hsa-miR-432-5p, hsa-miR-548a-3p, hsa-miR-589-3p, hsa-miR-655-3p } \\
\text { hsa-miR-6747-3p }\end{array}$ & $\begin{array}{l}\text { hsa-miR-548o-3p, hsa-miR-3184-5p, hsa-miR-27a-3p, hsa-miR-548f-3p, } \\
\text { hsa-let-7d-3p, hsa-miR-1293, hsa-miR-7641, hsa-miR-3607-3p, } \\
\text { hsa-miR-132-5p, hsa-miR-7-1-3p, hsa-miR-3158-3p, hsa-miR-361-5p, } \\
\text { hsa-miR-6723-5p, hsa-miR-1-3p, hsa-miR-148b-5p, hsa-miR-30b-5p, } \\
\text { hsa-miR-584-5p, hsa-miR-491-5p, hsa-miR-181b-3p, hsa-miR-574-3p, } \\
\text { hsa-miR-1303, hsa-let-7a-2-3p, hsa-miR-215-5p, hsa-miR-548q, } \\
\text { hsa-miR-629-3p, hsa-miR-1262, hsa-miR-107, hsa-miR-374a-5p, } \\
\text { hsa-let-7d-5p, hsa-miR-3688-3p, hsa-miR-4793-3p, hsa-miR-744-3p, } \\
\text { hsa-miR-4796-5p, hsa-miR-20a-3p, hsa-miR-122-5p, hsa-miR-6761-5p, } \\
\text { hsa-miR-6806-3p, hsa-miR-135b-5p, hsa-miR-4775, hsa-miR-450a-1-3p, } \\
\text { hsa-miR-142-5p, hsa-miR-378i, hsa-miR-7705, hsa-miR-548ay-3p, } \\
\text { hsa-miR-548j-3p, hsa-miR-1273g-3p, hsa-miR-130b-3p, hsa-miR-448, } \\
\text { hsa-miR-345-5p, hsa-let-7f-2-3p, hsa-miR-192-3p, hsa-miR-1249-5p, } \\
\text { hsa-miR-136-5p, hsa-miR-184, hsa-miR-3127-5p, hsa-miR-3163, } \\
\text { hsa-miR-3191-5p, hsa-miR-3654,hsa-miR-3691-5p, hsa-miR-3944-3p, } \\
\text { hsa-miR-4477b, hsa-miR-4787-3p, hsa-miR-485-5p, hsa-miR-578, } \\
\text { hsa-miR-6737-3p, hsa-miR-6843-3p }\end{array}$ \\
\hline
\end{tabular}




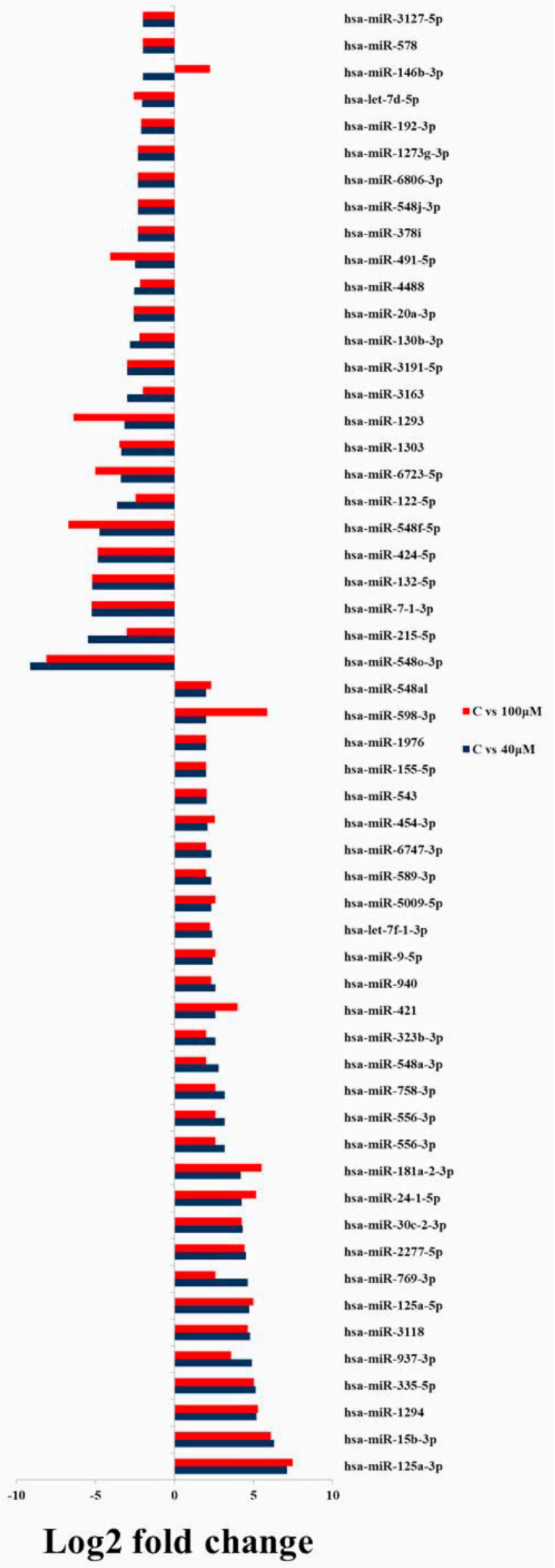

Figure 7. Common up- and down-regulated microRNAs between untreated control vs. 40 and the untreated control vs. $100 \mu \mathrm{M}$ EGCG treatments.

\subsection{Differential Expression Analysis of Putative Novel MicroRNA Sequences}

Heat maps were plotted to study the differential expression pattern of putative novel microRNAs. A complete putative microRNA profiling is shown in Figure 8. Significantly differentially expressed putative novel microRNAs are plotted as heat maps as shown in Figure 9a,b. Comparison of differential 
expression of putative novel microRNAs in the untreated control vs. 40, the untreated control vs. 100, and 40 vs. $100 \mu \mathrm{M}$ EGCG treatments showing greater than $2 \log 2$ fold change revealed 4 putative novel microRNAs differentially expressed in control vs. 40, 3 in control vs. 100, and 4 in 40 vs. $100 \mu \mathrm{M}$ EGCG treatment. In the untreated control vs. $40 \mu \mathrm{M}$ EGCG treatment, the putative novel microRNA TPP-A549-26 was up-regulated and three others namely TPP-A549-24, TPP-A549-27, and TPP-A549-28 were down-regulated. In addition, in the untreated control vs. $100 \mu \mathrm{M}$ EGCG treatment, putative novel microRNAs TPP-A549-32 and TPP-A549-29 were up-regulated and TPP-A549-30 and TPP-A549-31 were down-regulated (Table 3). The chromosomal location, precursor, and mature sequence details of greater than $2 \log 2$ fold change of putative novel microRNAs in the untreated control vs. 40 and the untreated control vs. $100 \mu$ M EGCG treatments are presented in Table 3. Three putative novel microRNAs namely TPP-A549-23, TPP-A549-24, and TPP-A549-25 were commonly differentially expressed in the untreated control vs. 40 and the untreated control vs. $100 \mu \mathrm{M}$ EGCG treatments (Figure 9). It was interesting to observe that putative novel microRNA TPP-A549-23 was up-regulated in the untreated control vs. $40 \mu \mathrm{M}$ EGCG treatment and was down-regulated in the untreated control vs. $100 \mu \mathrm{M}$ EGCG treatment (Figure 9c). 

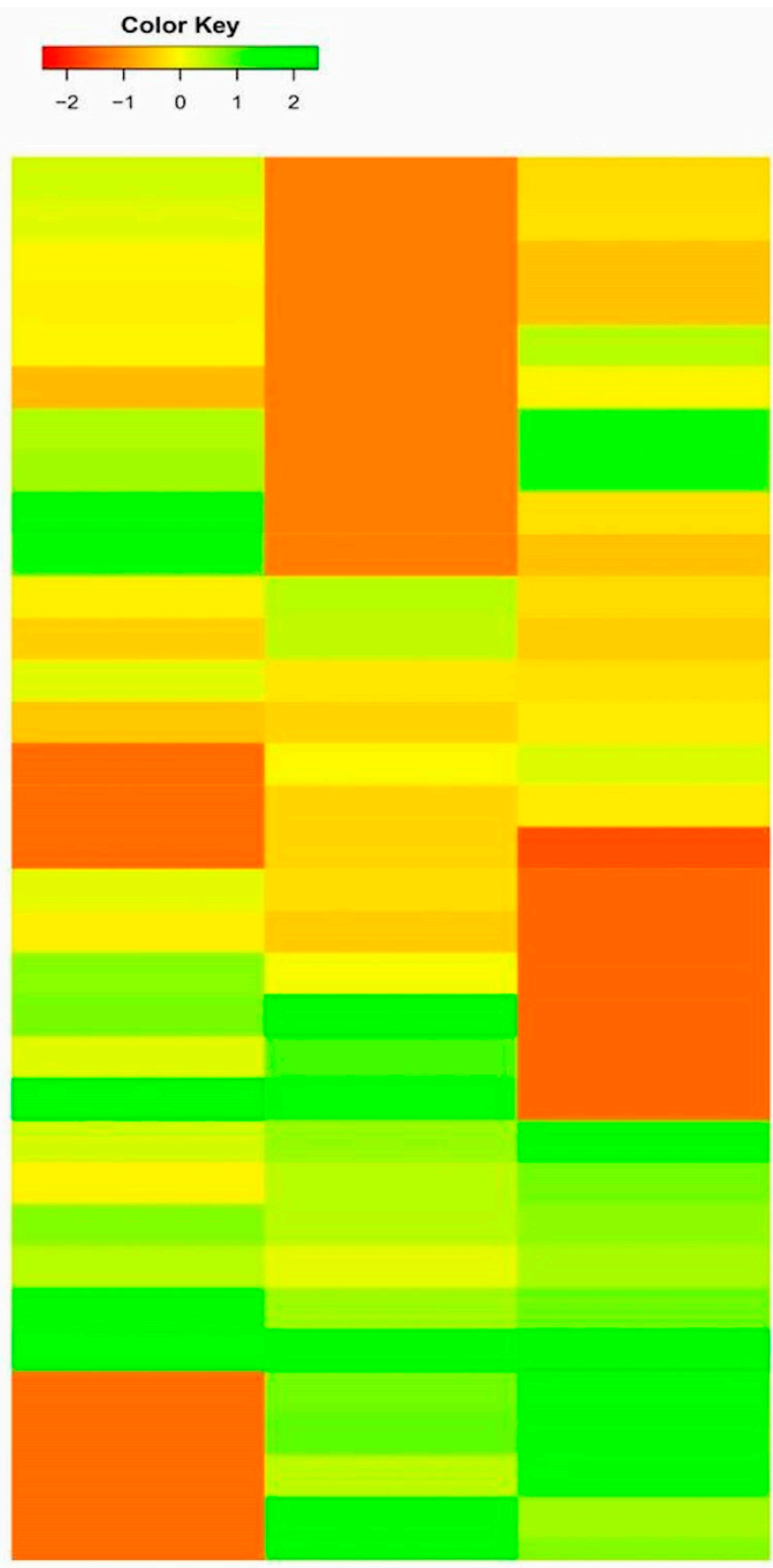

TIITTGCTGGAGGAGCATGGACT

GCTCCTGTGATGTTTCTGAGGCT

ACAGCTGTTGATCCACTCGGT

ATTAATATGTACTGACAAAGCGTA

AAATTTGGACAAATGAATGGAGTC

TGAGGAAGGGTTTGAGGCTG

GAGTGGATACAGATGCATGC

CAGGTCTTGTGGGTTCTGATG

TAGGTGGATTTGTGTGATGAGCT

CTTGCAATTACTTTTGCACCAACT

GTGGTGCGGTCTGATGTTIT

CTIGCAATTACTITTGCACTA

TGGAGGGACATTTGACTTCA

GTGAGGTAGTAGATTGTATAGTT

AGAAGTGCTACAGCTGCTGIT

CTTACCTTGGATTTGCTCTT

CCAGCCTACTGGAGGATAAGAGGA

GGCGGCCCCAGATCAGACGCC

ATGAGAATCTCCGGCGCCCA

GAAAAACCTGAGCTGATGGAC

CTCTGAACTGGGTAGATCTGTC

CAACTGITGGTGCATTTGGG

CTTAGAATTCCGATGCTGGGG

GAGGTAGAGCAAGGGCCCCT

CAGCTGCTGGATAATCATCCC

TCATTTGGGCAACAGGTTGGATTG

TCAGTGCACTACAGAACTTTGTC

AATAGGAATTGAGAGAAGTG

CAGATGGTTGCTGATCTGTGCA

AGCTGTAGTTGCTCCTGTGGAA

ATTTGGAAGCTCCTGTAGCTGAA

TTCAGGTTGAAGCTCTTGCACA

CTAGGACCATCACAGGCTGA

TGCGGGATAGATTTGTAGTCTGT

\section{Control $\quad 40 \mu \mathrm{M} \quad 100 \mu \mathrm{M}$}

Figure 8. The expression profiles of putative novel microRNAs in the untreated control, 40, and $100 \mu \mathrm{M}$ EGCG treatments. Color key- red: up-regulation, green: down-regulation and yellow: neutral expression. 

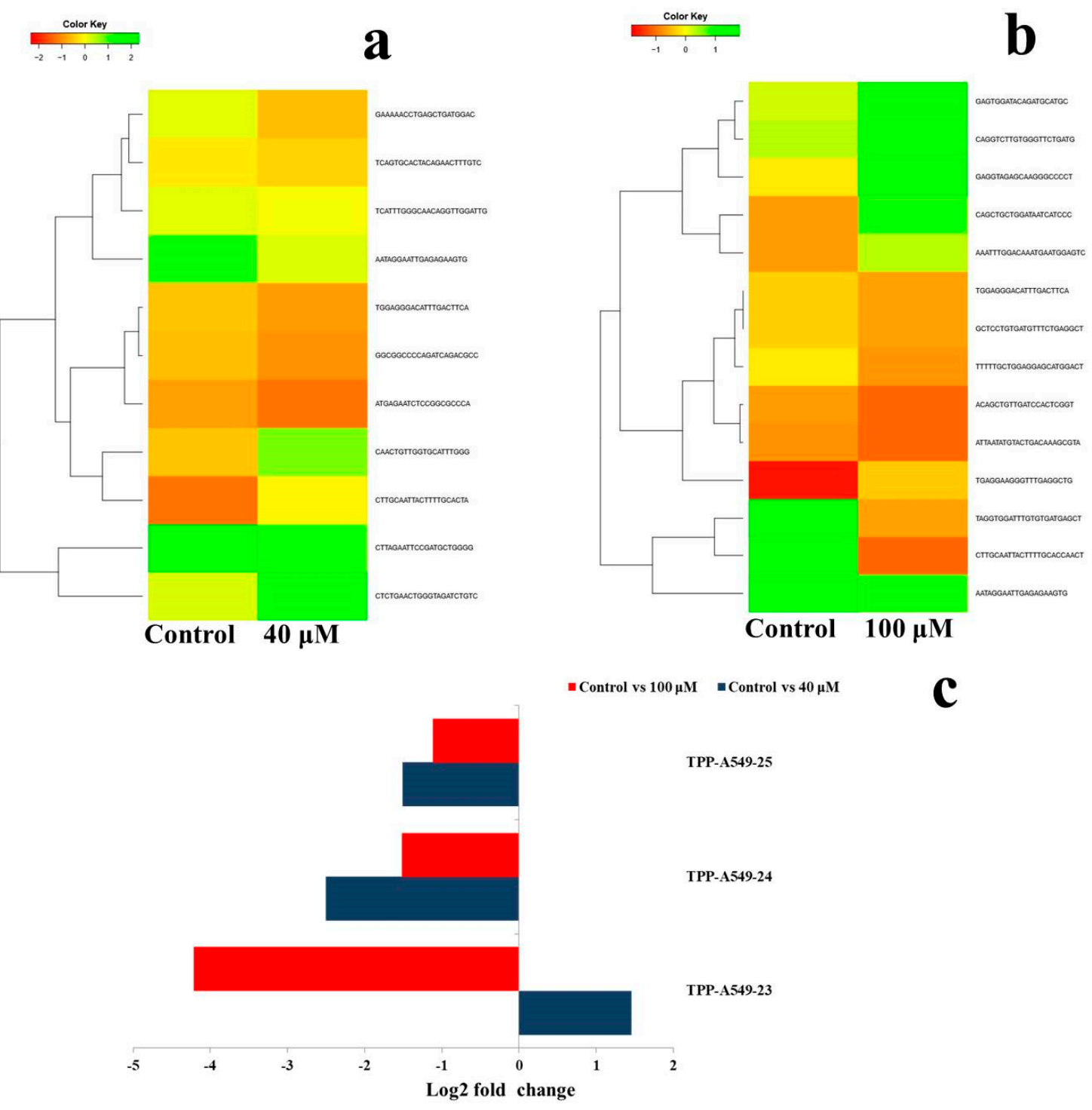

Figure 9. The differential expression pattern of putative novel microRNAs. (a) Untreated control vs. $40 \mu \mathrm{M}$ EGCG treatment; (b) Untreated control vs. $100 \mu \mathrm{M}$ EGCG treatments; (c) Log2 fold change of three putative novel microRNAs which were common in untreated control vs. 40 and untreated control vs. $100 \mu \mathrm{M}$ EGCG treatment. Color key- red: up-regulation, green: down-regulation and yellow: neutral expression. 
Table 3. Putative novel microRNA sequences with greater than $2 \log 2$ fold change in expression after 40 and $100 \mu \mathrm{M}$ EGCG treatments.

\begin{tabular}{|c|c|c|c|c|c|c|c|c|c|c|c|c|}
\hline \multirow{2}{*}{$\begin{array}{l}\text { Treatment } \\
\text { Compared }\end{array}$} & \multirow{2}{*}{$\begin{array}{c}\text { No. of } \\
\text { MicroRNAs }\end{array}$} & \multirow{2}{*}{$\begin{array}{c}\text { Putative } \\
\text { Novel Micro } \\
\text { RNA ID }\end{array}$} & \multirow{2}{*}{$\begin{array}{c}\text { Log2 Fold } \\
\text { Change }\end{array}$} & \multicolumn{2}{|c|}{ microRNA Sequences } & \multirow{2}{*}{$\begin{array}{l}\text { Chromosome } \\
\text { Number }\end{array}$} & \multirow{2}{*}{$\begin{array}{c}\text { Precursor } \\
\text { Sequence Start }\end{array}$} & \multirow{2}{*}{$\begin{array}{c}\text { Precursor } \\
\text { Sequence End }\end{array}$} & \multirow{2}{*}{$\begin{array}{c}\text { Mature } \\
\text { Sequence Start }\end{array}$} & \multirow{2}{*}{$\begin{array}{c}\text { Mature } \\
\text { Sequence End }\end{array}$} & \multirow{2}{*}{ Precursor Sequence } & \multirow{2}{*}{$\begin{array}{c}\text { MFE } \\
\text { (Kcal/mol) }\end{array}$} \\
\hline & & & & Up-Regulated & Down-Regulated & & & & & & & \\
\hline \multirow{4}{*}{$\begin{array}{l}\text { Control vs. } \\
\quad 40 \mu \mathrm{M}\end{array}$} & \multirow{4}{*}{4} & TPP-A549-26 & 2.52 & $\begin{array}{l}\text { CUCUGAACUGG } \\
\text { GUAGAUCUGUC }\end{array}$ & & 2 & $181,190,09$ & $181,190,98$ & $181,190,19$ & $181,190,40$ & $\begin{array}{l}\text { AACAGGGTAGCTCTGAACTGGGT } \\
\text { AGATCTGTCTGTCAAGAGGAACG } \\
\text { CTTCAGAAAGCTCAGGACTCTCC } \\
\text { TGCACAGGGCTTAGCCCACTG }\end{array}$ & -31.6 \\
\hline & & TPP-A549-24 & -2.5 & & $\begin{array}{l}\text { AAUAGGAAUU } \\
\text { GAGAGAAGUG }\end{array}$ & 4 & $107,162,755$ & $107,162,845$ & $107,162,765$ & $107,162,784$ & $\begin{array}{l}\text { CTCATGTTTTACTTCCTTTTCCTA } \\
\text { TTTTGTTACACTAGCTAGGGCTT } \\
\text { CTAGTAGAGAAATGAATAGGAAT } \\
\text { TGAGAGAAGTGATATTTTGGC }\end{array}$ & -27.5 \\
\hline & & TPP-A549-27 & -3.34 & & $\begin{array}{l}\text { CUUAGAAUUCC } \\
\text { GAUGCUGGGG }\end{array}$ & 22 & $267,618,39$ & $267,619,28$ & $267,618,98$ & $267,619,18$ & $\begin{array}{l}\text { AGATTCTGGACTTAGAATTCCGA } \\
\text { TGCTGGGGCCAGGCACAGTGGC } \\
\text { TCACACTGTAATCCCAGTACTTT } \\
\text { GGGAAGCCGAGGCGGGAGGAT }\end{array}$ & -28.8 \\
\hline & & TPP-A549-28 & -2.23 & & $\begin{array}{l}\text { GAAAAACCUGA } \\
\text { GCUGAUGGAC }\end{array}$ & 5 & $140,056,955$ & $140,057,037$ & $140,057,007$ & $140,057,027$ & $\begin{array}{l}\text { TCTAGATGATGAAAAACCTGAGCT } \\
\text { GATGGACAATGCCCGTAGTGTATT } \\
\text { TGTGATATGCAGCTCAGGTTTTT } \\
\text { CATTAATGTAA } \\
\end{array}$ & -36.3 \\
\hline \multirow{4}{*}{$\begin{array}{l}40 \text { vs. } \\
100 \mu \mathrm{M}\end{array}$} & \multirow{4}{*}{4} & TPP-A549-29 & 2.2 & $\begin{array}{l}\text { GAGGUAGAGC } \\
\text { AAGGGCCCCU }\end{array}$ & & 13 & $394,145,14$ & $394,146,06$ & $394,145,24$ & $394,145,43$ & $\begin{array}{l}\text { GGCTGGAGTTGAGGGTAGAGCAAG } \\
\text { GGCCCCTGCATTGGTGTTATCAG } \\
\text { TCTTCTGGGACTTCTGGGGTAC } \\
\text { TGTTTTCACTTCTTCTTCTTAT }\end{array}$ & -27.2 \\
\hline & & TPP-A549-30 & 2.6 & $\begin{array}{l}\text { UUCAGGUUGAA } \\
\text { GCUCUUGCACA }\end{array}$ & & 14 & $102,442,311$ & $102,442,392$ & $102,442,321$ & $102,442,342$ & $\begin{array}{l}\text { GGGATGAGTCTTCAGGTTGAAAGCT } \\
\text { CTTGCACAGCTGGCTCTCTCCTAG } \\
\text { CTGTGTAAGAACCTCTGGCCTGGG } \\
\text { TGCACAGCCA } \\
\end{array}$ & -38 \\
\hline & & TPP-A549-31 & -2.32 & & $\begin{array}{l}\text { CCAGCCUACUGGA } \\
\text { GGAUAAGAGGA }\end{array}$ & 8 & $102,442,311$ & $102,4423,92$ & $102,442,321$ & $102,442,342$ & $\begin{array}{c}\text { GGGATGAGTCTTCAGGTTGAAAGCT } \\
\text { CTTGCACAGCTGGCTCTCTCCTAG } \\
\text { CTGTGTAAGAACCTCTGGCCTGGG } \\
\text { TGCACAGCCA } \\
\end{array}$ & -38 \\
\hline & & TPP-A549-32 & -2.22 & & $\begin{array}{l}\text { CUAGGACCAU } \\
\text { CACAGGCUGA }\end{array}$ & 16 & $983,936,55$ & $983,937,34$ & $983,937,01$ & $983,937,24$ & $\begin{array}{c}\text { CTCTGGTTCCCCAGCCTACTGGAG } \\
\text { GATAAGAGGATATAAAGGTCTCTTA } \\
\text { TCCTCCAGTAGACTAGGGAGCCAG } \\
\text { AGCTGT }\end{array}$ & -53.7 \\
\hline \multirow{3}{*}{$\begin{array}{l}\text { Control vs. } \\
100 \mu \mathrm{M}\end{array}$} & \multirow{3}{*}{3} & TPP-A549-33 & 2.43 & $\begin{array}{l}\text { GAGGUAGAGC } \\
\text { AAGGGCCCCU }\end{array}$ & & 13 & $394,145,14$ & $394,146,06$ & $394,145,24$ & $394,145,43$ & $\begin{array}{l}\text { GGCTGGAGTTGAGGTAGAGCAA } \\
\text { GGGCCCCTGCATTTGGTGTTATC } \\
\text { AGTCTCTGGGACTTTCTTGGGG } \\
\text { TACTGTTTTCACTTCTTCTTCTTAT }\end{array}$ & -27.2 \\
\hline & & TPP-A549-23 & -4.2 & & $\begin{array}{l}\text { CUUGCAAUUACU } \\
\text { UUUGCACCAACU }\end{array}$ & 4 & $158,492,16$ & $158,493,08$ & $158,492,26$ & $158,492,49$ & $\begin{array}{l}\text { TAAATTATTAGGTTGGTTCAAAAA } \\
\text { TAATTGTGGTTTTGCCATTCCTTT } \\
\text { CGGTGGCAAAACTGCAATTACT } \\
\text { TTTGCACCAACTTAAATATATA }\end{array}$ & -37.4 \\
\hline & & TPP-A549-34 & -4.2 & & $\begin{array}{l}\text { UAGGUGGAUUUG } \\
\text { UGUGAUGAGCU }\end{array}$ & 9 & 195,7951 & 195,8044 & 195,7961 & 195,7983 & $\begin{array}{l}\text { AGGCCTGCCATAGGTGGATTTGTG } \\
\text { TGATGAGCTAAGGAGTTTCCAA } \\
\text { GCAGATATCATCTAATGGAAAAG } \\
\text { AGAACCAAGCACAAGCTCGGTCA }\end{array}$ & -25.5 \\
\hline
\end{tabular}




\section{7. $q R T-P C R$ Analysis of MicroRNAs}

The qRT-PCR analysis was performed to validate the NGS dataset. Hsa-miR-21-5p, hsa-miR-548o-5p, hsa-miR-181c, and hsa-miR-212-5p microRNAs were randomly selected for the study. qRT-PCR analysis showed 3.2, and $2.17 \log 2$ fold change in expression in the untreated control vs. $40 \mu \mathrm{M}$ EGCG treatment and 2.5 and $1.28 \log 2$ fold change in expression in the untreated control vs. $100 \mu \mathrm{M}$ EGCG treatment in hsa-miR-548o-5p and hsa-miR-181c, respectively. Furthermore, minimal differential $\log 2$ fold change expression of 0.31 and 0.03 in the untreated control vs. $40 \mu \mathrm{M}$ EGCG treatment and 0.76 and 0.6 in the untreated control vs. $100 \mu \mathrm{M}$ EGCG treatment was observed in hsa-miR-21-5p and hsa-miR-212-5p respectively (Figure 10).
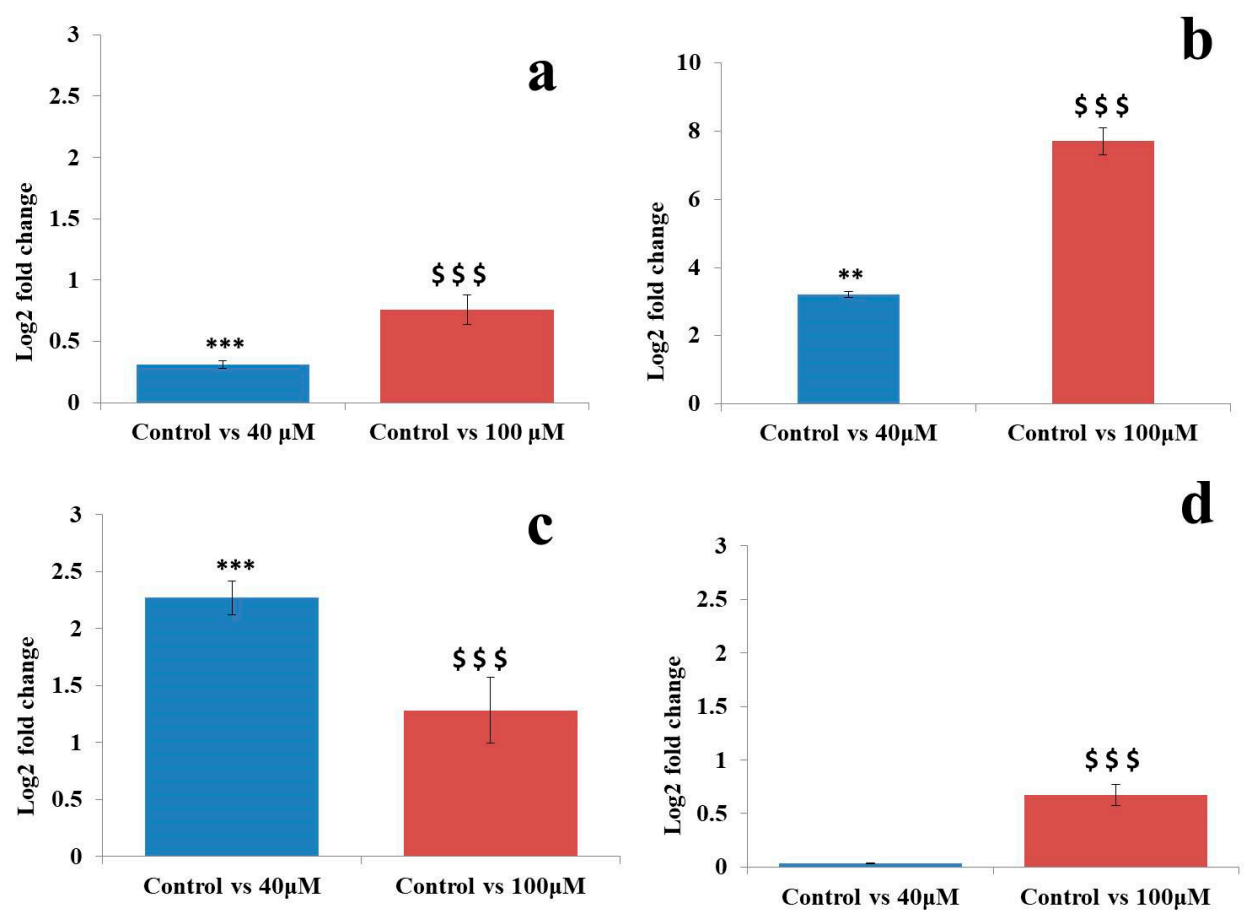

Figure 10. Validation of randomly selected known microRNAs by qRT-PCR. (a) Log2 fold change of miR-21-5p; (b) Log2 fold change of hsa-miR-548o-5p; (c) Log2 fold change of hsa-miR-181c; (d) Log2 fold change of hsa-miR-212-5p microRNAs. The significant difference between the untreated control vs. 40 and the untreated control vs. $100 \mu \mathrm{M}$ EGCG treatmts are indicated by '*' between untreated control and $40 \mu \mathrm{M}$ EGCG treatment, and ' $\$$ ' between untreated control and $100 \mu \mathrm{M}$ EGCG treatment. Significance levels of $p<0.001\left(^{(* * * \prime} ; ' \$ \$ \$\right.$ '), $p<0.01\left(^{(* * \prime)}\right.$ are denoted by repetition of these symbols.

In microRNA sequencing data, an up-regulation of hsa-miR-181c by 3.51 and $1.5 \log 2$ fold was observed in the untreated control vs. 40 and the untreated control vs. $100 \mu \mathrm{M}$ EGCG treatments, respectively. Furthermore, a significant down-regulation of hsa-miR-548o-5p by 9 and $8 \log 2$ fold change was noted in the untreated control vs. 40 and the untreated control vs. $100 \mu \mathrm{M}$ EGCG treatments respectively (Figure 11). About 0.1 and $0.5 \log 2$ fold change of hsa-miR-212-3p was observed in untreated control vs. $40 \mu \mathrm{M}$ EGCG treatment. In hsa-miR-181c, the log2 fold change of 0.15 and 1.5 was noted in untreated control vs. $100 \mu \mathrm{M}$ EGCG treatment. A comparative fold change between the qRT-PCR and sequencing dataset supports each other. Therefore, q-RT PCR results validate the present NGS dataset. 

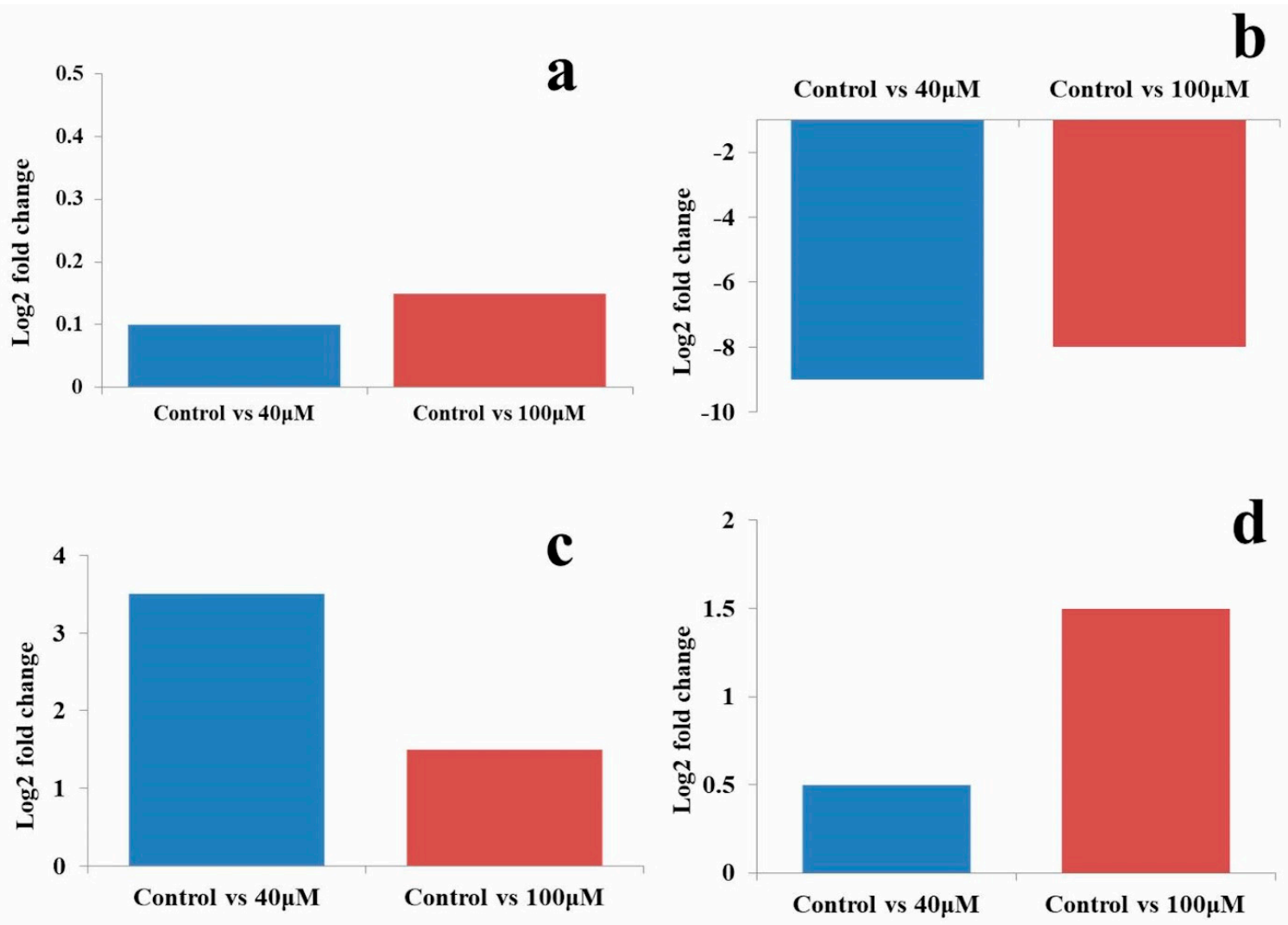

Figure 11. Log2 fold change obtained by NGS dataset in the untreated control vs. 40 and the untreated control vs. 100 MM EGCG treatments. (a) miR-21-5p; (b) hsa-miR-548o-5p; (c) hsa-miR-181c; (d) hsa-miR-212-5p microRNAs.

A qRT-PCR analysis of the putative novel miRNA TPP-A549-23 showed 1.95 and $4.2 \log 2$ fold change of expression in the untreated control vs. 40 and the untreated control vs. $100 \mu \mathrm{M}$ EGCG treatments respectively (Figure 12). This fold change was compared with the log2 fold change obtained from the NGS data analysis. This sequence was up-regulated by $1.45 \log 2$ fold change in the untreated control vs. $40 \mu \mathrm{M}$ EGCG treatment and was down-regulated by 4.21 -fold change in the untreated control vs. $100 \mu \mathrm{M}$ EGCG treatment (Figure 12).

It was observed that $\log 2$ fold change obtained in sequencing dataset was similar to the $\log 2$ fold change obtained from the qRT-PCR analysis. Furthermore, the present study also validates a novel microRNA sequence obtained in the NGS dataset. This qRT-PCR analysis attests to our computational analysis of NGS data. 

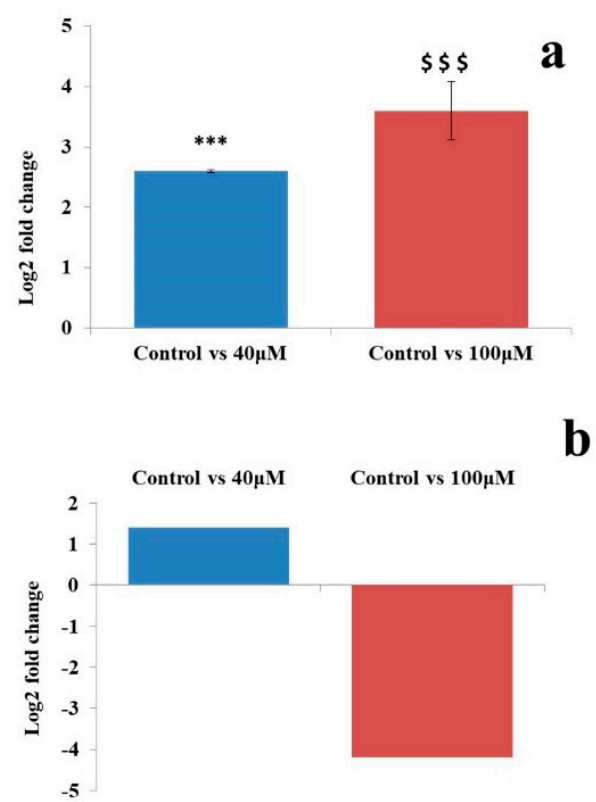

Figure 12. Validation of putative of novel microRNA TPP-A549-23 by qRT PCR. (a) Log2 fold change from qRT-PCR; (b) Log2 fold change from NGS dataset. Significant difference is indicated by ${ }^{* \prime \prime}$ between the untreated control and $40 \mu \mathrm{M}$ EGCG treatment and ' $\$$ ' between the untreated control and $100 \mu \mathrm{M}$ EGCG treatment. Significance levels of $p<0.001\left({ }^{(* * * \prime ;} ;\right.$ ' $\$ \$ \$$ ') are denoted by repetition of these symbols.

\subsection{KEGG and PANTHER Pathway Enrichment of Targets of Validated microRNAs}

The high precision target prediction for hsa-miR-548o-5p, hsa-miR-181c, hsa-miR-212-5p, and hsa-miR-21-5p was carried out using TargetScan and miRDB target computational prediction software. Default cut off values were used for gene target prediction. KEGG and PANTHER pathway analysis were carried out using the Database for Annotation, Visualization and Integrated Discovery (DAVID) and the pathways were shortlisted. Pathway analysis for microRNAs was evaluated and common pathways predicted between TargetScan and miRDB are presented in Figure 13. Pathways in cancer, MAPK, regulation of actin cytoskeleton, wnt signaling, ErbB signaling, B-cell and T-cell receptor signaling, and long-term potentiation were the most significant pathways obtained in KEGG pathway analysis (Figure 13a,c,e,g). In addition, Ras signaling, angiogenesis, FGF signaling, wnt signaling, FGF, and PDGF (Platelet-derived growth factor) signaling pathway genes were reported in PANTHER pathway analysis (Figure 13b,d,f,h). 

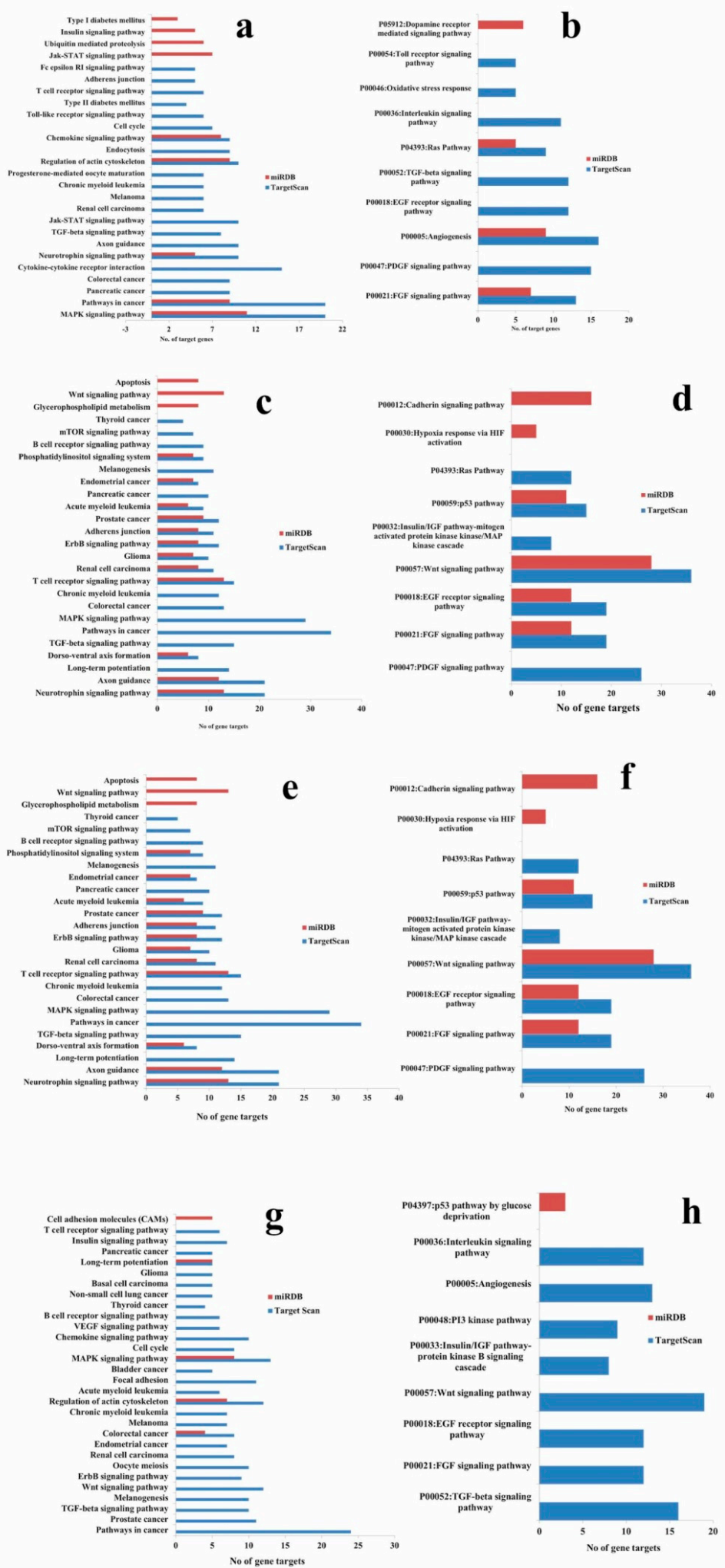

Figure 13. KEGG and PANTHER pathway enrichment of targets of validated microRNAs. (a) KEGG pathway analysis of hsa-miR-21-5p; (b) PANTHER pathway analysis of hsa-miR-21-5p; (c) KEGG pathway analysis of hsa-miR-548o-5p; (d) PANTHER pathway analysis of hsa-miR-548o-5p; (e) KEGG pathway analysis of hsa-miR-181c; (f) PANTHER pathway analysis of hsa-miR-181c; (g) KEGG pathway analysis of hsa-miR-212-5p; (h) PANTHER pathway analysis of hsa-miR-212-5p. 
Furthermore, KEGG pathway analysis showed the MAPK signaling pathway as the target for hsa-miR-21-5p, hsa-miR-548o-5p, hsa-miR-181c, and hsa-miR-212-5p microRNAs (Table 4). Our analysis with PANTHER pathway did not show any common pathway among the microRNAs. Ras signaling pathway, wnt pathway, angiogenesis, p53, and EGF (Epidermal growth factor) receptor signaling pathway were the most significant pathways predicted by TargetScan and miRDB target list. MiRanda software was used for target prediction and pathway analysis of putative novel microRNA sequences. The common pathways found in the target prediction were wnt, angiogenesis, p-53, PI3K, and MAPK signaling pathways. The putative novel microRNA TPP-A549-23 targeted FOPNL (FGFR1OP N-Terminal Like), ACVR1C (Activin A Receptor Type 1C), and CD38 (Cyclin-Dependent Kinase Inhibitor 2B) genes. Furthermore, the putative novel microRNA TPP-A549-24 targeted RBM18 (RNA Binding Motif Protein 18) and CDKN2B (Cyclin-Dependent Kinase Inhibitor 2A). In addition, APC, RADGEF2 (Rap Guanine Nucleotide Exchange Factor 2), CNNM2 [Cyclin and CBS (cystathionine-beta-synthase) Domain Divalent Metal Cation Transport Mediator 2] CORO2A (Cronin 2A), SEPT9 (Septin 9), RAPGEF2 (Rap Guanine Nucleotide Exchange Factor 2), JAK1 (Janus Kinase 1), and WDR19 (WD Repeat Domain 19) were noted to be potential target genes for the putative novel microRNA TPP-A549-25. The pathway analysis showed that the putative microRNAs could play an important role in cell cycle proliferation, MAPK, Hedgehog, FOXO, and TGF-beta signaling cascade. We believe that these predicted putative novel microRNA sequences play a major role in cancer proliferation and metastasis. 
Table 4. List of common pathways in TargetScan and mirDB.

\begin{tabular}{|c|c|c|c|c|}
\hline MicroRNAs & KEGG Pathways Common in TargetScan and miRDB & $\begin{array}{l}\text { PANTHER Pathways Common in } \\
\text { TargetScan and miRDB }\end{array}$ & $\begin{array}{l}\text { KEGG Pathway Common in } \\
\text { all the MicroRNAs }\end{array}$ & $\begin{array}{l}\text { PANTHER Pathways } \\
\text { Common in all the } \\
\text { microRNAs }\end{array}$ \\
\hline Hsa-miR-21-5p & $\begin{array}{l}\text { MAPK signaling pathway, } \\
\text { Regulation of actin cytoskeleton, } \\
\text { Chemokine signaling pathway, } \\
\text { Pathways in cancer, } \\
\text { Neurotrophin signaling pathway }\end{array}$ & $\begin{array}{l}\text { P00021: FGF signaling pathway, } \\
\text { P00005: Angiogenesis, } \\
\text { P04393: Ras Pathway }\end{array}$ & & \\
\hline Hsa-miR-548o-5p & $\begin{array}{l}\text { Small cell lung cancer, Colorectal cancer, Pancreatic cancer, } \\
\text { Renal cell carcinoma, T cell receptor signaling pathway, } \\
\text { the Hedgehog signaling pathway, Long-term potentiation, } \\
\text { TGF-beta signaling pathway, ErbB signaling pathway, } \\
\text { Endometrial cancer, B cell receptor signaling pathway, } \\
\text { Acute myeloid leukemia, } \\
\text { Glioma, Non-small cell lung cancer, } \\
\text { MAPK signaling pathway, Axon guidance, Apoptosis, } \\
\text { Wnt signaling pathway }\end{array}$ & P00057: Wnt signaling pathway & & \\
\hline Hsa-miR-181c & $\begin{array}{l}\text { Neurotrophin signaling pathway, } \\
\text { Axon guidance, } \\
\text { Long-term potentiation, } \\
\text { Dorso-ventral axis formation, } \\
\text { TGF-beta signaling pathway, } \\
\text { Pathways in cancer, } \\
\text { MAPK signaling, pathway } \\
\text { Colorectal cancer, } \\
\text { Chronic myeloid leukemia, } \\
\text { T cell receptor signaling pathway, } \\
\text { Renal cell carcinoma, } \\
\text { Glioma, } \\
\text { ErbB signaling pathway, } \\
\text { Adherens junction, } \\
\text { Prostate cancer, } \\
\text { Acute myeloid leukemia, } \\
\text { Pancreatic cancer, } \\
\text { Endometrial cancer, } \\
\text { Phosphatidylinositol signaling system }\end{array}$ & $\begin{array}{l}\text { P00021: FGF signaling pathway, } \\
\text { P00018: EGF receptor signaling pathway, } \\
\text { P00057: Wnt signaling pathway } \\
\text { P00032: Insulin/IGF pathway-mitogen } \\
\text { activated protein kinase/MAP kinase cascade, } \\
\text { P00059: p53 pathway }\end{array}$ & MAPK signaling pathway & Nil \\
\hline Hsa-miR-212-5p & $\begin{array}{l}\text { Colorectal cancer, } \\
\text { Regulation of actin cytoskeleton, } \\
\text { MAPK signaling pathway, } \\
\text { Long-term potentiation }\end{array}$ & Nil & & \\
\hline
\end{tabular}




\section{Discussion}

To evaluate the changes of EGCG induced microRNA expression in A549 cells, an established in vitro model of human lung adenocarcinoma, and next-generation sequencing analysis were employed. The dose of EGCG used in the present study was decided based on cell cycle analysis and previous literature. EGCG is capable of causing G0/G1 phase arrest in many cancer cell lines including A549 [37-40]. The present cell cycle data analysis showed G0/G1 phase arrest at $40 \mu \mathrm{M}$ EGCG treatment. Kweon et al. [41] reported that out of eight cell lines they studied, A549 showed no sign of apoptosis and was highly resistant even at $100 \mu$ M EGCG treatment. As much as $85 \%$ of the cell viability was sustained for $72 \mathrm{~h}$ at $40 \mu \mathrm{M}$ EGCG treatment. We observed browning of the medium above $100 \mu$ M EGCG treatment. Previous data suggested that $\mathrm{O}_{2}{ }^{-}$and quinones generation in the cell culture medium occurs due to the auto-oxidation properties of EGCG [42,43]. Hence, in the present study, we have chosen $40 \mu \mathrm{M}$ and $100 \mu \mathrm{M}$ EGCG concentrations.

Nine hundred and fifty-nine microRNAs out of 1881 microRNAs (50.9\%) reported in miRbase (as per Feb 2018) were expressed in A549 cells. Some microRNAs did not show differential expression while certain other microRNAs were significantly influenced by EGCG treatment. Therefore, EGCG dependent microRNA profiling was studied according to the three independent criteria namely log2 expression analysis, differentially up- and down-regulated microRNAs and putative gene targets of the microRNAs.

In the present study, the next-generation raw data was analyzed for greater than $2 \log 2$ fold change of expression in known and putative novel microRNA sequences. In known microRNA dataset, out of 959 microRNAs, $115(11.9 \%)$ and $134(13.9 \%)$ microRNAs in 40 and $100 \mu$ M EGCG treatments respectively exhibited greater than $2 \log 2$ fold change of expression (Table 2).In the putative novel microRNA dataset, out of 208 microRNAs, $4(1.9 \%)$ and $3(1.4 \%)$ microRNAs in 40 and $100 \mu \mathrm{M}$ EGCG treatments respectively showed differential expression above $2 \log 2$ fold change (Table 3). Interestingly, some microRNAs namely hsa-miR-125a-3p, hsa-miR-15b-3p, and hsa-miR-548av-3p in untreated control vs. $40 \mu \mathrm{M}$ EGCG treatment and hsa-miR-125a-3p, hsa-miR-500a-3p, hsa-miR-7706, and hsa-miR-15b-3p in untreated control vs. $100 \mu$ M EGCG treatment exhibited greater than 6 $\log 2$ fold change of expression. These observations strongly support that EGCG can modulate multiple microRNAs. The putative novel microRNAs TPP-A549-14, TPP-A549-24, TPP-A549-27, and TPP-A549-28 in untreated control vs. $40 \mu \mathrm{M}$ EGCG treatment and TPP-A549-32, TPP-A549-23, and TPP-A549-33 in untreated control vs. $100 \mu \mathrm{M}$ EGCG treatment showed more than 2 log2 fold change of expression.

Interestingly, the top 10 up-expressed microRNAs namely, hsa-miR-21-5p, hsa-let-7i-5p, hsa-miR-100-5p, hsa-miR-27b-3p, hsa-miR-151a-3p, hsa-miR-148a-3p, hsa-miR-30a-5p, hsa-miR-192-5p, hsa-miR-3529-3p, and hsa-miR-30d-5p were similar in untreated control and EGCG treatments. The hsa-miR-21 is the most commonly up-expressed microRNA in human cancers [44-46]. This microRNA is the first one to be named as "oncomir" [47] and was found to highly up-expressed in 540 clinical samples from cancer patients [48]. Yang et al. [49] reported significant up-regulation of hsa-miR-21 in NSCLC patients. In addition, they also reported that inhibition of hsa-miR-21 expression reduced cell proliferation, migration, and invasion in A549 cell. Up-expression of hsa-miR-21 in untreated control A549 cells further attests to our NGS data. Furthermore, due to treatment with EGCG, no significant down-regulation of hsa-miR-21 was observed in differential expression analysis of our NGS data (Figure 11a) which is validated by qRT-PCR analysis (Figure 10a). It is well known that hsa-miR-21 is an oncogene which plays an important role in programmed cell death and targeting apoptosis [50] but, in the present study no such apoptosis was observed on EGCG treatments. Therefore, we hypothesize that EGCG treatment has no modulatory effect on hsa-miR-21. Therefore, the present data support the non-apoptotic effect of EGCG in A549 cells. Another clinical study validated the chemoresistance role of hsa-mir-21 on platinum-based chemotherapy [51]. The persistent expression of hsa-miR-21 in untreated control and EGCG-treated A549 cells in the present study is supported by the clinical microarray data validated by Fujita et al. [51]. 
Let-7i, a member of the let-7 family, is an oncogenic driver of NSCLC. Increased expression of let-7i in the present study supports the statistically significant clinical study conducted on NSCLC [52]. Furthermore, a microarray analysis of primary lung cancer tumors and non-cancerous lung tissues revealed down-regulation of hsa-miR-181c [53]. In addition, Fujita et al. [51] reported the significance of hsa-miR-181c. It was observed that patients which did not respond to platinum-based chemotherapy had elevated hsa-miR-181c expression as compared to the well responsive patients. This study thus validated the chemoresistant role of hsa-miR-181c [51]. In the present study, EGCG treatment elevated the expression of hsa-miR-181c (Figure 9c). Moreover, this data was validated by qRT-PCR analysis (Figure 8c), where we found a significant up-regulation of hsa-miR-181c by 2.17 and $1.28 \log 2$ fold change by 40 and $100 \mu \mathrm{M}$ EGCG treatments, respectively.

The direction of $\log 2$ fold change (up- or down-regulation) was analyzed in microRNA expression. About 53 and 69 microRNAs were up-regulated after 40 and $100 \mu$ M EGCG treatments, respectively. Hsa-miR-125a-3p was highly up-regulated with a log2 fold change of 7.12 and 7.47 in 40 and $100 \mu \mathrm{M}$ EGCG treatments, respectively. Jiang et al. [54] showed an inverse relationship between hsa-miR-125a-3p with invasion and metastasis. Furthermore, it was marked that hsa-miR-125a-3p was down-regulated in NSCLC [54]. Significant up-regulation of hsa-miR-125a-3p was observed with EGCG treatment in A549 cells. As it was established that EGCG inhibits cell proliferation in A549 cells by causing G0/G1 phase arrest (Figure 1), up-regulation of signature microRNA hsa-miR-125a-3p attests to the role of EGCG in inhibiting cell proliferation and invasion.

Hsa-miR-548o-3p was significantly down-regulated by 9.12 and $8.12 \log 2$ fold change in 40 and $100 \mu \mathrm{M}$ EGCG treatments, respectively. Mir-548 family consist of 68 microRNAs and is the largest, and poorly conserved primate-specific gene family [55]. The reports on one of the members of mir-548 family, hsa-miR-548c-3p, revealed it as a functional biomarker in prostate and gastric cancer progression [54,56]. However, the exact function of hsa-miR-548o-3p in A549 cells is still unknown, but evidence supports the up-regulation of the mir-548 family in cancer progression. We noted down-regulation of hsa-miR-548o-3p by EGCG treatments (Figures $8 \mathrm{~b}$ and $9 \mathrm{~b}$ ) which indicated it as a potential biomarker for cancer progression.

Hsa-miR-212 is a tumor suppressor microRNA which negatively regulates anti-apoptotic protein PED/PEA-15 [57]. A study by Jiang et al. [58] validated the biological role of hsa-miR-212/132 in A549 cells. Up-regulation of hsa-mir-212 blocked proliferation and migration and was observed to cause cell cycle arrest by modulating p21 and cyclin D1 expression [58]. An analysis of our NGS data supported the biological role of hsa-mir-212. We observed no significant expression of hsa-mir-212 in untreated control A549 cells. The lower expression of hsa-miR-212 signifies the cancerous property of A549 cells. In addition, negligible differential expression was observed in the EGCG-treated A549 cells. This indicated the fact that A549 cells are resistant to EGCG treatment.

Notably, a significant modulation in hsa-miR-146b-3p was observed with EGCG treatment. Down-regulation of hsa-miR-146b-3p was observed with $40 \mu \mathrm{M}$ EGCG treatment and a notable up-regulation was noticed in $100 \mu \mathrm{M}$ EGCG treatment (Figure 6). A clinical study elucidated a down-regulation of hsa-miR-146b-3p in breast cancer tissues. Further findings revealed that hsa-miR-146b-3p's over-expression suppressed migration, invasion, metastasis, and growth in breast cancer cell lines [59]. The principal findings on the role of hsa-miR-146-3p in untreated control A549 cells revealed the association of its lower expression with early-stage NSCLC recurrence [60]. Functional role of hsa-miR-146b-3p is still unexplored, but our data indicated its important role in tumor suppression.

The goal of this study was to identify putative novel microRNA sequences in A549 cells and their potential differences in expression between untreated control and EGCG treatments. We reported, 4, 3 , and 4 putative novel microRNA sequences in untreated control vs. 40, untreated control vs. 100, and 40 vs. $100 \mu \mathrm{M}$ EGCG treatments respectively (Table 3). Only three putative microRNA sequences were persistently present between the untreated control vs. 40 and the untreated control vs. $100 \mu \mathrm{M}$ EGCG treatments (Figure 9c). To validate the log2 fold change of the putative microRNA sequences, 
qRT-PCR analysis was done. The expression analysis of the putative novel microRNA TPP-A549-23 was done using qRT-PCR analysis. We observed similar log2 fold change between the untreated control vs. 40 and the untreated control vs. $100 \mu \mathrm{M}$ EGCG treatments (Figure 8). The NGS dataset and validation confirmed the significant expression of this putative novel microRNA. Further validation of the sequence is required.

TargetScan and miRDB databases identified approximately 1200 potential target genes of microRNAs (data not shown). Next, we have used DAVID web-based gene ontology and pathway prediction software for further analysis. KEGG pathway and PANTHER pathway analysis were carried out with both the gene targets obtained by TargetScan and miRDB.

Target prediction and pathway analysis of hsa-miR-21-5p, hsa-miR-548o-5p, hsa-miR-181c, and hsa-miR-212-5p revealed MAPK, pathways in cancer, Ras, FGF, wnt, and T-cell receptor signaling pathways as the most significant pathways in our study (Figure 13). Further analysis showed that these microRNAs commonly target the MAPK signaling pathway. The present study supports the functional role of hsa-miR-21-5p, hsa-miR-548o-5p, hsa-miR-181c, and hsa-miR-212-5p. Significant expression of these microRNAs in untreated control and EGCG-treated A549 cells demonstrated their important role in regulating cell proliferation.

For target prediction of putative novel microRNAs, we used miRanda 3.3a. Approximately 30 target genes were predicted for which pathway analysis was done. The putative novel microRNA sequences showed wnt, angiogenesis, p-53, PI3K, and MAPK signaling pathways as major targeting pathways. The common putative novel microRNAs differentially expressed between the control and EGCG treatments namely TPP-A549-23, TPP-A549-24, and TPP-A549-25 were shown to target cell cycle, MAPK, Hedgehog, FOXO, and TGF-beta signaling pathways. FOPNL, ACVR1C, CD38. RBM18, CDKN2B, APC, RADGEF2, CNNM2, CORO2A, SEPT9, RAPGEF2, JAK1, and WDR19 genes were the potential targets for these putative novel microRNA sequences. These putative novel microRNA sequences showed an important role in cell cycle modulation and MAPK signaling cascade.

In summary, we demonstrated the important role of EGCG in modulating microRNA expression profiling. Substantial evidence from previous studies have provided an important role of EGCG in inhibiting cell proliferation in lung cancer, but its mechanistic insights on microRNA profiling remain incompletely understood. However, our results provided a useful approach for better understanding of EGCG induced microRNA modulation. Furthermore, exploration with transfection and human lung cancer tissue should be performed to validate the microRNA profiling and their predicted targets.

\section{Materials and Methods}

\subsection{Cell Culture and Treatment}

Human non-small cell lung cancer A549 cells (purchased from National Centre for Cell Science, Pune, India) was cultured in F12 Ham-K medium containing 1X antibiotic-antimycotic solution and $10 \%$ FBS (Himedia, India) at $37{ }^{\circ} \mathrm{C}$ with $95 \%$ humidified atmosphere and $5 \% \mathrm{CO}_{2}$. For cell cycle analysis, A549 cells were seeded in $60 \mathrm{~mm}$ cell culture dishes for $24 \mathrm{~h}$ in the F12-Ham-K medium. The cells were further treated with 20, 40, 80, 100, and $150 \mu$ M EGCG (Sigma Chemical Co. St. Louis, Missouri, United States, USA). After $24 \mathrm{~h}$, cells were harvested and re-suspended in $100 \mu \mathrm{L}$ PBS. The cells were fixed by drop-wise addition of $8-10 \mathrm{~mL} 75 \%$ ice-cold ethanol, continuous stirring, and incubating on ice for $15 \mathrm{~min}$. After centrifugation, the cells were re-suspended in $200 \mu \mathrm{L}$ PBS containing $0.04 \mathrm{mg} / \mathrm{mL}$ propidium iodide and $0.1 \mathrm{mg} / \mathrm{mL}$ RNase and was incubated for $30 \mathrm{~min}$ at $37^{\circ} \mathrm{C}$ in dark. Cell cycle analysis was carried out on BD FACSVerse ${ }^{\mathrm{TM}}$ flow cytometer (ThermoFisher Scientific, Massachusetts, United States.

\subsection{Next-Generation Sequencing}

A549 cells were treated with EGCG at the concentration of 40 and $100 \mu \mathrm{M}$. Untreated cells were used as a control. Cells were harvested for RNA extraction after $24 \mathrm{~h}$ of culture. Total RNA 
was extracted from the untreated and treated A549 cells using TRizol reagent (Takara Bio Inc., Kusatsu, Japan) following the manufacturer's protocol. The small RNA library construction and deep sequencing were carried out at AgriGenome Labs Pvt. Ltd., Kochi, Kerala, India. NEBNext®Multiplex Small RNA Library Prep Set for Illumina was used for library construction. The raw counts and the normalized files were submitted in NCBI (National Center for Biotechnology Information) by accession number GSE110514.

\subsection{Classification and Differential Expression Analysis of MicroRNAs}

The human genome (GRCh38) was used for the mapping of microRNA reads using Bowtie. Known miRNAs were identified using miRBase-21 database based on miRNA sequence similarity approach. The sequences were checked for other ncRNA contamination (rRNA, tRNA, snRNA, snoRNA, and piRNA). The novel microRNA prediction was evaluated by Mireap_0.22b [36]. Furthermore, secondary hairpin structures were predicted using mfold [61]. Expressed reads for each microRNAs were calculated and DESseq R software package was used for differential expression analysis. Differentially expressed microRNAs in control vs. 40, control vs. 100 and 40 vs. $100 \mu$ M EGCG treatments were determined by their expression in each sample [62]. The expressed reads in untreated control, 40, and $100 \mu \mathrm{M}$ EGCG treatments were used to calculate the log2 fold change of expression between untreated control vs. 40, untreated control vs. 100, and 40 vs. $100 \mu \mathrm{M}$ EGCG treatments.

\subsection{Validation of MicroRNAs}

To validate the expression of some of the significant known and putative novel microRNAs, total microRNA was isolated using miRNeasy Mini Kit (Qiagen Sciences, USA) from untreated control and EGCG-treated (40 and $100 \mu \mathrm{M}$ ) A549 cells. First-strand cDNA was synthesized using miScript PCR starter kit (Qiagen Sciences, Germantown, MD, USA) and SYBR green (nucleic acid strain) was used for qRT-PCR (ABI Prism 7000, ThermoFisher Scientific, Massachusetts, United States). The log2 fold change was calculated using the $\triangle \Delta C T$ method [63].

The comparative analysis of qRT-PCR and NGS was done to validate the microRNA expression profile obtained by NGS. The log2 fold change obtained by qRT-PCR of the known microRNAs namely miR-548o-3p, miR-212, miR-125a, and miR-181c was calculated and compared with the log2 fold change obtained in the NGS sequencing data. The log2 fold change of the putative novel microRNA TPP-A549-23 obtained by qRT-PCR and NGS was also compared for the sequence validation.

\subsection{KEGG and PANTHER Pathway Enrichment of Targets of Validated MicroRNAs}

The target prediction for known microRNAs was performed using TargetScan and miRDB [56] and the pathway analysis was done by The Database for Annotation, Visualization and Integrated Discovery (DAVID V 6.7). In addition, target prediction of the putative novel microRNA sequences was carried out using miRanda software [64].

\subsection{Statistical Analysis}

Statistical analysis was performed with one-way analysis of variance (ANOVA). The experimental data was represented as mean $\pm \mathrm{SD}$. The results were considered significant when $p<0.001, p<0.01$ or $p<0.05$.

\section{Conclusions}

We used NGS to study a complete microRNA profiling of A549 cells and identified 958, 944 and 935 known microRNAs in the untreated control, 40, and $100 \mu \mathrm{M}$ EGCG treatments, respectively. The oncogenic microRNAs were highly up-expressed in the untreated control and EGCG-treated A549 cells which are the part of broadly conserved let-7, hsa-miR-21, and hsa-miR-30 seed families. The up-expressions of these oncogene microRNAs with EGCG treatment indicated resistance 
character of A549 cells for EGCG. The differential expression analysis of microRNAs identified highly up-regulated hsa-miR-125a-3p and highly down-regulated hsa-miR-548o-3p in the untreated control vs. 40 and the untreated control vs. $100 \mu \mathrm{M}$ EGCG treatments, respectively. A similar log2 fold change in the comparative analysis between NGS and qRT-PCR of randomly selected known and putative novel microRNAs validated the NGS data. Our data indicated EGCG as an effective natural compound which regulates microRNA profile in A549 cells. This study also attested the modulation of microRNAs by EGCG which regulates cell cycle and inhibits cell proliferation and metastasis. KEGG and PANTHER pathway analysis revealed the MAPK pathway as the most potent targeted pathway by EGCG modulated microRNAs. The findings explored an important role of EGCG in microRNA regulation, which targets MAPK cascade. Furthermore, the putative novel microRNA sequences reported in this study can be a novel approach towards the microRNA targeting gene therapies.

Author Contributions: A.K.A.M. was responsible for the concept and designing of this study. V.B. performed the NGS analysis and validation of microRNAs in A549 cell line. V.B. and A.K.A.M. drafted the manuscript. A.K.A.M.

Funding: The authors are thankful to the NTRF, Kolkata for financial support.

Acknowledgments: The authors are thankful to the National Tea Research Foundation (NTRF) for funding and the management of VIT University for providing research facilities. The authors also acknowledge the support of Genotypic Technology Limited, Bangalore for processing of NGS data, Ramalingam B of National Institute of Research in Tuberculosis, Chennai, and Narender Kumar of the University of Cambridge, UK for raw data submission to NCBI.

Conflicts of Interest: The authors declare no conflict of interest.

\section{References}

1. Min, K.; Kwon, T.K. Anticancer effects and molecular mechanisms of epigallocatechin-3-gallate. Integr. Med. Res. 2014, 3, 16-24. [CrossRef] [PubMed]

2. Singh, T.; Katiyar, S.K. Green tea polyphenol, (-)-epigallocatechin-3-gallate, induces toxicity in human skin cancer cells by targeting beta-catenin signaling. Toxicol. Appl. Pharmacol. 2013, 273, 418-424. [CrossRef] [PubMed]

3. Zhou, D.-H.; Wang, X.; Feng, Q. EGCG enhances the efficacy of cisplatin by downregulating hsa-miR-98-5p in NSCLC A549 cells. Nutr. Cancer 2014, 66, 636-644. [CrossRef] [PubMed]

4. Jankun, J.; Selman, S.H.; Swiercz, R.; Skrzypczak-Jankun, E. Why drinking green tea could prevent cancer. Nature 1997, 387, 561. [CrossRef] [PubMed]

5. Shimizu, M.; Deguchi, A.; Lim, J.T.E.; Moriwaki, H.; Kopelovich, L.; Weinstein, I.B. (-)-Epigallocatechin gallate and polyphenon $\mathrm{E}$ inhibit growth and activation of the epidermal growth factor receptor and human epidermal growth factor receptor-2 signaling pathways in human colon cancer cells. Clin. Cancer Res. 2005, 11, 2735-2746. [CrossRef] [PubMed]

6. García, V.; Lara-chica, M.; Cantarero, I.; Sterner, O.; Marco, A.; Muñoz, E. Galiellalactone induces cell cycle arrest and apoptosis through the ATM/ATR pathway in prostate cancer cells. Oncotarget 2015, 7. [CrossRef] [PubMed]

7. Mukhtar, H.; Ahmad, N. Tea polyphenols: Prevention of cancer and optimizing health. Am. J. Clin. Nutr. 2000, 71, 1698S-1702S; discussion 1703S-1704S. [CrossRef]

8. Yang, C.S.; Maliakal, P.; Meng, X. Inhibition of carcinogenesis by tea. Annu. Rev. Pharmacol. Toxicol. 2002, 42, 25-54. [CrossRef]

9. Jemal, A.; Siegel, R.; Ward, E.; Hao, Y.; Xu, J.; Thun, M.J. Cancer Statistics. CA Cancer J. Clin. 2009, 59, $225-249$. [CrossRef]

10. Torre, L.A.; Bray, F.; Siegel, R.L.; Ferlay, J.; Lortet-Tieulent, J.; Jemal, A. Global cancer statistics, 2012. CA Cancer J. Clin. 2015, 65, 87-108. [CrossRef]

11. Molina, J.R.; Yang, P.; Cassivi, S.D.; Schild, S.E.; Adjei, A.A. Non-small cell lung cancer: Epidemiology, risk factors, treatment, and survivorship. Mayo Clin. Proc. 2008, 83, 584-594. [CrossRef]

12. Sun, S.; Schiller, J.H.; Gazdar, A.F. Lung cancer in never smokers-A different disease. Nat. Rev. Cancer 2007, 7, 778-790. [CrossRef] [PubMed] 
13. Condoluci, A.; Mazzara, C.; Zoccoli, A.; Pezzuto, A.; Tonini, G. Impact of smoking on lung cancer treatment effectiveness: A review. Future Oncol. 2016, 12, 2149-2161. [CrossRef] [PubMed]

14. Sini, C.; Tuzi, A.; Rossi, G.; Russo, A.; Pezzuto, A. Acquired resistance in oncogene-addicted non-small-cell lung cancer. Future Oncol. 2018, 14, 29-40. [CrossRef] [PubMed]

15. Pezzuto, A.; Manicone, M.; Scaini, M.C.; Ricci, A.; Mariotta, S.; Zamarchi, R.; Rossi, E. What information could the main actors of liquid biopsy provide?-A representative case of non-small cell lung cancer (NSCLC). J. Thorac. Dis. 2018, 10, E570-E576. [CrossRef] [PubMed]

16. Stahlhut, C.; Slack, F.J. MicroRNAs and the cancer phenotype: Profiling, signatures and clinical implications. Genome Med. 2013, 5, 111. [CrossRef] [PubMed]

17. Choudhury, Y.; Tay, F.C.; Lam, D.H.; Sandanaraj, E.; Tang, C.; Ang, B.T.; Wang, S. Attenuated adenosine-to-inosine editing of microRNA-376a * promotes invasiveness of glioblastoma cells. J. Clin. Investig. 2012, 122, 4059-4076. [CrossRef]

18. Dhillon, A.S.; Hagan, S.; Rath, O.; Kolch, W. MAP kinase signalling pathways in cancer. Oncogene 2007, 26, 3279-3290. [CrossRef]

19. Sarris, E.G.; Saif, M.W.; Syrigos, K.N. The biological role of PI3K pathway in lung cancer. Pharmaceuticals 2012, 5, 1236-1264. [CrossRef]

20. Thorpe, L.M.; Yuzugullu, H.; Zhao, J.J. PI3K in cancer: Divergent roles of isoforms, modes of activation and therapeutic targeting. Nat. Rev. Cancer 2014, 15, 7. [CrossRef]

21. Thomas, S.J.; Snowden, J.A.; Zeidler, M.P.; Danson, S.J. The role of JAK/STAT signalling in the pathogenesis, prognosis and treatment of solid tumours. Br. J. Cancer 2015, 113, 365-371. [CrossRef] [PubMed]

22. Zhan, T.; Rindtorff, N.; Boutros, M. Wnt signaling in cancer. Oncogene 2017, 36, 1461-1473. [CrossRef]

23. De Luca, A.; Maiello, M.R.; D'Alessio, A.; Pergameno, M.; Normanno, N. The RAS/RAF/MEK/ERK and the PI3K/AKT signalling pathways: Role in cancer pathogenesis and implications for therapeutic approaches. Expert Opin. Ther. Targets 2012, 16 (Suppl. 2), S17-S27. [CrossRef] [PubMed]

24. McCubrey, J.A.; Steelman, L.S.; Chappell, W.H.; Abrams, S.L.; Wong, E.W.T.; Chang, F.; Lehmann, B.; Terrian, D.M.; Milella, M.; Tafuri, A.; et al. Roles of the Raf/MEK/ERK pathway in cell growth, malignant transformation and drug resistance. Biochim. Biophys. Acta Mol. Cell Res. 2007, 1773, 1263-1284. [CrossRef] [PubMed]

25. Taylor, M.A.; Wappett, M.; Delpuech, O.; Brown, H.; Chresta, C.M. Enhanced MAPK signaling drives ETS1-mediated induction of miR-29b leading to downregulation of TET1 and changes in epigenetic modifications in a subset of lung SCC. Oncogene 2016, 35, 4345-4357. [CrossRef] [PubMed]

26. Shi, L.; Middleton, J.; Jeon, Y.J.; Magee, P.; Veneziano, D.; Laganà, A.; Leong, H.S.; Sahoo, S.; Fassan, M.; Booton, R.; et al. KRAS induces lung tumorigenesis through microRNAs modulation article. Cell Death Dis. 2018, 9. [CrossRef]

27. Bartel, D.P. MicroRNAs: Genomics, Biogenesis, Mechanism, and Function. Cell 2004, 116, 281-297. [CrossRef]

28. Krol, J.; Loedige, I.; Filipowicz, W. The widespread regulation of microRNA biogenesis, function and decay. Nat. Rev. Genet. 2010, 11, 597. [CrossRef]

29. Friedman, R.C.; Farh, K.K.-H.; Burge, C.B.; Bartel, D.P. Most mammalian mRNAs are conserved targets of microRNAs. Genome Res. 2009, 19, 92-105. [CrossRef]

30. Calin, G.A.; Sevignani, C.; Dumitru, C.D.; Hyslop, T.; Noch, E.; Yendamuri, S.; Shimizu, M.; Rattan, S.; Bullrich, F.; Negrini, M.; et al. Human microRNA genes are frequently located at fragile sites and genomic regions involved in cancers. Proc. Natl. Acad. Sci. USA 2004, 101, 2999-3004. [CrossRef]

31. Liu, J.; Jennings, S.F.; Tong, W.; Hong, H. Next generation sequencing for profiling expression of miRNAs: Technical progress and applications in drug development. J. Biomed. Sci. Eng. 2011, 4, 666-676. [CrossRef] [PubMed]

32. Duncavage, E.; Goodgame, B.; Sezhiyan, A.; Govindan, R.; Pfeifer, J. Use of MicroRNA expression levels to predict outcomes in resected stage i non-small cell lung cancer. J. Thorac. Oncol. 2010, 5, 1755-1763. [CrossRef] [PubMed]

33. Markou, A.; Sourvinou, I.; Vorkas, P.A.; Yousef, G.M.; Lianidou, E. Clinical evaluation of microRNA expression profiling in non small cell lung cancer. Lung Cancer 2013, 81, 388-396. [CrossRef] [PubMed]

34. Wang, Y.; Chen, J.; Lin, Z.; Cao, J.; Huang, H.; Jiang, Y.; He, H.; Yang, L.; Ren, N.; Liu, G. Role of deregulated microRNAs in non-small cell lung cancer progression using fresh-frozen and formalin-fixed, paraffin-embedded samples. Oncol. Lett. 2016, 11, 801-808. [CrossRef] [PubMed] 
35. Gallach, S.; Calabuig-Fariñas, S.; Jantus-Lewintre, E.; Camps, C. MicroRNAs: Promising new antiangiogenic targets in cancer. Biomed. Res. Int. 2014, 2014. [CrossRef] [PubMed]

36. Ji, Z.; Wang, G.; Zhang, C.; Xie, Z.; Liu, Z.; Wang, J. Identification and function prediction of novel MicroRNAs in Laoshan dairy goats. Asian-Australas. J. Anim. Sci. 2013, 26, 309-315. [CrossRef] [PubMed]

37. Ma, Y.-C.; Li, C.; Gao, F.; Xu, Y.; Jiang, Z.-B.; Liu, J.-X.; Jin, L.-Y. Epigallocatechin gallate inhibits the growth of human lung cancer by directly targeting the EGFR signaling pathway. Oncol. Rep. 2014, 31, 1343-1349. [CrossRef] [PubMed]

38. Gupta, S.; Hussain, T.; Mukhtar, H. Molecular pathway for (-)-epigallocatechin-3-gallate-induced cell cycle arrest and apoptosis of human prostate carcinoma cells. Arch. Biochem. Biophys. 2003, 410, 177-185. [CrossRef]

39. Masuda, M.; Suzui, M.; Weinstein, I.B. Effects of epigallocatechin-3-gallate on growth, epidermal growth factor receptor signaling pathways, gene expression, and chemosensitivity in human head and neck squamous cell carcinoma cell lines. Clin. Cancer Res. 2001, 7, 4220-4229.

40. Ahmad, N.; Cheng, P.; Mukhtar, H. Cell cycle dysregulation by green tea polyphenol epigallocatechin-3-gallate. Biochem. Biophys. Res. Commun. 2000, 275, 328-334. [CrossRef]

41. Kweon, M.-H.; Saleem, M.; Mukhtar, H. Constitutive overexpression of Nrf2-dependent HO-1 confers resistance to apoptosis by EGCG in human lung adenocarcinoma A549 cells. Cancer Res. 2006, 66, 1149.

42. Hou, Z.; Sang, S.; You, H.; Lee, M.J.; Hong, J.; Chin, K.V.; Yang, C.S. Mechanism of action of (-)-epigallocatechin-3-gallate: Auto-oxidation- dependent inactivation of epidermal growth factor receptor and direct effects on growth inhibition in human esophageal cancer KYSE 150 cells. Cancer Res. 2005, 65, 8049-8056. [CrossRef] [PubMed]

43. Hong, J.; Lu, H.; Meng, X.; Colon, H.-H.; Ryu, J.; Hara, Y.; Yang, C.S. Stability, Cellular Uptake, Biotransformation, and Efflux of Tea Polyphenol (-)-Epigallocatechin-3-Gallate in HT-29 Human Colon Adenocarcinoma Cells Stability, Cellular Uptake, Biotransformation, and Efflux of Tea Polyphenol. Cancer Res. 2002, 62, 7241-7246.

44. Xue, X.; Liu, Y.; Wang, Y.; Meng, M.; Wang, K.; Zang, X.; Zhao, S.; Sun, X.; Cui, L.; Pan, L.; et al. MiR-21 and MiR-155 promote non-small cell lung cancer progression by downregulating SOCS1, SOCS6, and PTEN. Oncotarget 2016, 7, 84508-84519. [CrossRef]

45. Markou, A.; Zavridou, M.; Lianidou, E.S. MiRNA-21 as a novel therapeutic target in lung cancer. Lung Cancer Targets Ther. 2016, 7, 19-27. [CrossRef]

46. Li, C.; Yin, Y.; Liu, X.; Xi, X.; Xue, W.; Qu, Y. Non-small cell lung cancer associated microRNA expression signature: Integrated bioinformatics analysis, validation and clinical significance. Oncotarget 2017, 8 , 24564-24578. [CrossRef]

47. Medina, P.P.; Nolde, M.; Slack, F.J. OncomiR addiction in an in vivo model of microRNA-21-induced pre-B-cell lymphoma. Nature 2010, 467, 86-90. [CrossRef]

48. Volinia, S.; Calin, G.A.; Liu, C.-G.; Ambs, S.; Cimmino, A.; Petrocca, F.; Visone, R.; Iorio, M.; Roldo, C.; Ferracin, M.; et al. A microRNA expression signature of human solid tumors defines cancer gene targets. Proc. Natl. Acad. Sci. USA 2006, 103, 2257-2261. [CrossRef]

49. Yang, Y.; Meng, H.; Peng, Q.; Yang, X.; Gan, R.; Zhao, L.; Chen, Z.; Lu, J.; Meng, Q.H. Downregulation of microRNA-21 expression restrains non-small cell lung cancer cell proliferation and migration through upregulation of programmed cell death 4. Cancer Gene Ther. 2015, 22, 23-29. [CrossRef]

50. Buscaglia, L.E.B.; Li, Y. Apoptosis and the target genes of microRNA-21. Chin. J. Cancer 2011, 30, 371-380. [CrossRef]

51. Fujita, Y.; Yagishita, S.; Hagiwara, K.; Yoshioka, Y.; Kosaka, N.; Takeshita, F.; Fujiwara, T.; Tsuta, K.; Nokihara, H.; Tamura, T.; et al. The clinical relevance of the miR-197/CKS1B/STAT3-mediated PD-L1 network in chemoresistant non-small-cell lung cancer. Mol. Ther. 2015, 23, 717-727. [CrossRef] [PubMed]

52. Fassina, A.; Cappellesso, R.; Fassan, M. Classification of non-small cell lung carcinoma in transthoracic needle specimens using microRNA expression profiling. Chest 2011, 140, 1305-1311. [CrossRef] [PubMed]

53. Yanaihara, N.; Caplen, N.; Bowman, E.; Seike, M.; Kumamoto, K.; Yi, M.; Stephens, R.M.; Okamoto, A.; Yokota, J.; Tanaka, T.; et al. Unique microRNA molecular profiles in lung cancer diagnosis and prognosis. Cancer Cell 2006, 9, 189-198. [CrossRef] [PubMed] 
54. Jiang, L.; Huang, Q.; Zhang, S.; Zhang, Q.; Chang, J.; Qiu, X.; Wang, E. Hsa-miR-125a-3p and hsa-miR-125a-5p are downregulated in non-small cell lung cancer and have inverse effects on invasion and migration of lung cancer cells. BMC Cancer 2010, 10. [CrossRef] [PubMed]

55. Liang, T.; Guo, L.; Liu, C. Genome-wide analysis of mir-548 gene family reveals evolutionary and functional implications. J. Biomed. Biotechnol. 2012, 2012. [CrossRef] [PubMed]

56. Rane, J.K.; Scaravilli, M.; Ylipää, A.; Pellacani, D.; Mann, V.M.; Simms, M.S.; Nykter, M.; Collins, A.T.; Visakorpi, T.; Maitland, N.J. MicroRNA expression profile of primary prostate cancer stem cells as a source of biomarkers and therapeutic targets. Eur. Urol. 2015, 67, 7-10. [CrossRef] [PubMed]

57. Incoronato, M.; Garofalo, M.; Urso, L.; Romano, G.; Quintavalle, C.; Zanca, C.; Iaboni, M.; Nuovo, G.; Croce, C.M.; Condorelli, G. miR-212 increases tumor necrosis factor-related apoptosis-inducing ligand sensitivity in non-small cell lung cancer by targeting the antiapoptotic protein PED. Cancer Res. 2010, 70, 3638-3646. [CrossRef]

58. Jiang, X.; Chen, X.; Chen, L.; Ma, Y.; Zhou, L.; Qi, Q.; Liu, Y.; Zhang, S.; Luo, J.; Zhou, X. Upregulation of the miR-212/132 cluster suppresses proliferation of human lung cancer cells. Oncol. Rep. 2015, 33, 705-712. [CrossRef]

59. Xiang, W.; Wu, X.; Huang, C.; Wang, M.; Zhao, X.; Luo, G. PTTG1 regulated by miR-146a-3p promotes bladder cancer migration, invasion, metastasis and growth. Oncotarget 2016, 8, 1-15. [CrossRef]

60. Patnaik, S.K.; Kannisto, E.; Mallick, R.; Yendamuri, S. Overexpression of the lung cancer-prognostic miR-146b microRNAs has a minimal and negative effect on the malignant phenotype of A549 lung cancer cells. PLoS ONE 2011, 6. [CrossRef]

61. Zuker, M. Mfold web server for nucleic acid folding and hybridization prediction. Nucl. Acids Res. 2003, 31, 3406-3415. [CrossRef] [PubMed]

62. Anders, S.; Huber, W. Differential expression analysis for sequence count data. Genome Biol. 2010, 11, 1-12. [CrossRef] [PubMed]

63. Livak, K.J.; Schmittgen, T.D. Analysis of relative gene expression data using real-time quantitative PCR and the $2^{-\Delta \Delta C T}$ method. Methods 2001, 25, 402-408. [CrossRef] [PubMed]

64. Riffo-Campos, Á.L.; Riquelme, I.; Brebi-Mieville, P. Tools for sequence-based miRNA target prediction: What to choose? Int. J. Mol. Sci. 2016, 17, 1987. [CrossRef] [PubMed]

Sample Availability: Samples of the compounds are not available from the authors.

(C) 2019 by the authors. Licensee MDPI, Basel, Switzerland. This article is an open access article distributed under the terms and conditions of the Creative Commons Attribution (CC BY) license (http://creativecommons.org/licenses/by/4.0/). 